\title{
Do Leading Indicators Lead Peaks More Than Troughs?*
}

\author{
Richard Paap Rene Segers ${ }^{\dagger} \quad$ Dick van Dijk \\ Econometric Institute and Tinbergen Institute \\ Erasmus University Rotterdam
}

ECONOMETRIC Institute Report EI 2007-08

March 20, 2007

\begin{abstract}
We develop a formal statistical approach to investigate the possibility that leading indicator variables have different lead times at business cycle peaks and troughs. For this purpose, we propose a novel Markov switching vector autoregressive model, where economic growth and leading indicators share a common Markov process determining the state, but such that their cycles are non-synchronous with the non-synchronicity varying across the different regimes. An empirical application to monthly US industrial production (IP) and The Conference Board's Composite Index of Leading Indicators (CLI) for the period 1959-2004 shows that on average the CLI leads IP by more than seven months at peaks, but only by three and a half months at troughs. In terms of timeliness, the CLI is therefore most useful for signalling oncoming recessions. Furthermore, we find that allowing for asymmetric lead times leads to improved real-time dating of business cycle peaks and troughs and more accurate forecasts of turning points and IP growth.
\end{abstract}

Keywords: Business Cycles, Leading Indicators, Markov Switching, Bayesian Inference, Real-time data

JEL Classification Codes: C11, C32, C51, E32.

*We thank participants of the conference on "Nonlinearity and the Business Cycle", Washington University at St. Louis, August 2004, the Econometric Society European Meeting in Vienna, August 2006, and the 17th (EC) ${ }^{2}$ Meeting in Rotterdam, December 2006, for helpful comments. We thank Ataman Ozyildirim at The Conference Board for providing the real-time CLI data.

$\dagger$ Corresponding author. Econometric Institute, Erasmus University Rotterdam, P.O. Box 1738, NL-3000 DR Rotterdam, The Netherlands. Tel.: +31-10-4081315, fax: +31-10-4089162. E-mail addresses: paap@few.eur.nl (R. Paap), rsegers@few.eur.nl (R. Segers), djvandijk@few.eur.nl (D. van Dijk) 


\section{Introduction}

Reliable leading indicators of the business cycle are of great importance for policymakers, firms, and investors. It is therefore not surprising that economists set out on an intensive quest for such leading indicators, ever since the initial attempts of Mitchell and Burns (1938) for the US economy. This research has provided much insight into the construction, use, and evaluation of leading indicators, see Marcellino (2006) for a recent survey.

Reliability of a leading indicator variable includes aspects such as consistency and timeliness. By consistency we refer to the property that a leading indicator should systematically give an accurate indication of the future course of the economy and should not produce false turning point signals too frequently, for example. Timeliness means that in order to be useful, a leading indicator variable should have a considerable lead time with respect to business cycle turning points. Most of the currently popular leading indicator variables are believed to have a lead time between six and eighteen months. At the same time, it appears to be the case that many of these variables have a considerably longer lead time at business cycle peaks than at troughs. For example, the Composite Index of Leading Indicators (CLI) currently published by The Conference Board has led cyclical downturns in the economy by eight to twenty months, and upturns by one to ten months during the post-World War II period (The Conference Board, 2001).

In this paper, we develop a formal approach to investigate whether leading indicator variables have different lead times at peaks and troughs. For this purpose, we propose a novel Markov switching vector autoregressive model, where economic growth and leading indicator variables share a common cycle determined by a single Markov process, but such that their regime-switching is not exactly synchronous with the length of the displacement, or lead/lag time varying across the different regimes. We follow a Bayesian approach for estimation of the model parameters, with posterior results being obtained through flexible Markov Chain Monte Carlo techniques. The advantage of Bayesian analysis of the model is that it allows us to treat the lead/lag times as unknown parameters. We can then use their posterior distributions to conduct statistical inference on the asymmetry of the lead/lag structure at peaks and troughs. 
We provide an empirical application involving monthly US industrial production (IP) and The Conference Board's CLI over the period 1959-2004. We find that on average the CLI leads IP by more than seven months at peaks, but only by three and a half months at troughs. This suggests that, in terms of timeliness, the CLI is most useful for signalling oncoming recessions. The posterior results provide convincing evidence in favor of the presence of a non-synchronous common cycle with asymmetric lead times. The Bayes' factor relative to an alternative specification with equal lead times at cyclical downturns and upturns is very large. The same applies to models with synchronous cycles and with independent cycles in the different variables. In addition, the CLI is more consistent and more timely in terms of signaling oncoming recessions when embedded in the general model specification. In order to examine the practical usefulness of allowing for asymmetric lead times we conduct a business cycle dating and forecasting exercise for the period from October 1987 to July 2004, using real-time data for both the CLI and IP. We find that allowing for asymmetric lead times leads to more timely and precise identification of peaks and troughs for the 1990-1991 and 2001 recessions, as well as more accurate out-of-sample forecasts of turning points as well as IP growth rates.

The paper is organized as follows. In Section 2, we introduce our novel Markov switching vector autoregressive model. In addition, we describe (nested) alternative specifications, which allow for a non-synchronous common cycle but with identical lead times at all possible regime switches, for a synchronous common cycle, and for independent cycles. To facilitate interpretation of the models, we focus on the bivariate case, where both economic growth (or the coincident indicators) and the leading indicators are represented by a single variable. We provide details of the Bayesian approach for parameter estimation and inference in Section 3. In Section 4 we discuss the empirical results based on estimating the different model specifications over the complete sample period. In Section 5, we consider the real-time performance of the alternative cycle representations in terms of identifying peaks and troughs, and forecasting turning points and IP growth. Finally, we conclude in Section 6 . 


\section{Model specification}

Our point of departure is the assumption that the cycles in both output (or another measure of aggregate economic activity) and the leading indicator consist of two regimes (although extensions to multiple regimes are possible) labeled 'recession' and 'expansion', which are characterized by different mean growth rates of these variables. To make this precise, let $y_{1, t}$ and $y_{2, t}$ denote the growth rates of output and the leading indicator, respectively, in period $t$. Consider the unobserved binary random variables $s_{1, t}$ and $s_{2, t}$, where $s_{j, t}$ takes the value 0 in case $y_{j, t}$ is in expansion and 1 in case $y_{j, t}$ is in the recession regime. The mean growth rate conditional on the state $s_{j, t}$ is denoted as $\mu_{j, s_{j, t}} \equiv \mathrm{E}\left[y_{j, t} \mid s_{j, t}\right]$, for $j=1,2$, where typically $\mu_{j, 1}<0<\mu_{j, 0}$ such that recessions and expansions correspond to periods with negative and positive average growth, respectively. The properties of $s_{1, t}$ and $s_{2, t}$ determine the relationship between the cyclical behavior of economic activity and leading indicators, and we return to these in detail below. For the moment it is sufficient to say that they are assumed to be homogeneous first-order Markov processes. Finally, assuming first-order autoregressive dynamics in the demeaned growth rates, we arrive at the specification

$$
\begin{aligned}
& y_{1, t}-\mu_{1, s_{1, t}}=\phi_{1,1}\left(y_{1, t-1}-\mu_{1, s_{1, t-1}}\right)+\phi_{1,2}\left(y_{2, t-1}-\mu_{2, s_{2, t-1}}\right)+\varepsilon_{1, t} \\
& y_{2, t}-\mu_{2, s_{2, t}}=\phi_{2,1}\left(y_{1, t-1}-\mu_{1, s_{1, t-1}}\right)+\phi_{2,2}\left(y_{2, t-1}-\mu_{2, s_{2, t-1}}\right)+\varepsilon_{2, t},
\end{aligned}
$$

where

$$
\left(\begin{array}{l}
\varepsilon_{1, t} \\
\varepsilon_{2, t}
\end{array}\right) \sim \text { i.i.d. } N\left(\left(\begin{array}{l}
0 \\
0
\end{array}\right),\left(\begin{array}{ll}
\sigma_{1,1} & \sigma_{1,2} \\
\sigma_{2,1} & \sigma_{2,2}
\end{array}\right)\right),
$$

and $\varepsilon_{1, t_{1}}$ and $\varepsilon_{2, t_{1}}$ are independent of $s_{1, t_{2}}$ and $s_{2, t_{2}}$ for all $t_{1}$ and $t_{2}$. We may write this model in vector notation as

$$
\left(Y_{t}-\mathcal{M}_{\mathcal{S}_{t}}\right)=\Phi\left(Y_{t-1}-\mathcal{M}_{\mathcal{S}_{t-1}}\right)+\mathcal{E}_{t}, \text { with } \mathcal{E}_{t} \sim N(0, \Sigma)
$$

where

$$
Y_{t}=\left(\begin{array}{c}
y_{1, t} \\
y_{2, t}
\end{array}\right), \quad \mathcal{S}_{t}=\left(\begin{array}{c}
s_{1, t} \\
s_{2, t}
\end{array}\right), \quad \mathcal{M}_{\mathcal{S}_{t}}=\left(\begin{array}{c}
\mu_{s_{1, t}} \\
\mu_{s_{2, t}}
\end{array}\right), \quad \text { and } \quad \mathcal{E}_{t}=\left(\begin{array}{l}
\varepsilon_{1, t} \\
\varepsilon_{2, t}
\end{array}\right)
$$

The Markov switching vector autoregressive (MS-VAR) model in (3) obviously needs to be completed by specifying the exact dynamic properties of $s_{1, t}$ and $s_{2, t}$. 
We consider four different specifications, which allow for varying degrees of interrelation between the cycles in economic growth and in the leading indicator. First, an extreme standpoint would be to assume that these cycles are completely independent. In this case, the state vectors $s_{1, t}$ and $s_{2, t}$ can be defined as two independent first-order two-state homogeneous Markov processes with transition probabilities

$$
\operatorname{Pr}\left[s_{j, t}=0 \mid s_{j, t-1}=0\right]=p_{j} \quad \text { and } \quad \operatorname{Pr}\left[s_{j, t}=1 \mid s_{j, t-1}=1\right]=q_{j}, \quad j=1,2 .
$$

Second, the other extreme would be to assume that the variables $y_{1, t}$ and $y_{2, t}$ share a common business cycle, which is obtained by imposing

$$
s_{2, t}=s_{1, t}, \quad \text { for all } t
$$

As a consequence, a single underlying Markov process with transition probabilities $p$ and $q$ can be used to model the business cycle. We refer to Krolzig (1997), Paap and van Dijk (2003), and Chauvet and Hamilton (2006) for extensive treatments of this model specification.

Third, a more subtle approach, as proposed in Hamilton and Perez-Quiros (1996), is to assume that, although economic growth and leading indicators share the same business cycle, the cycle of the leading indicators leads or lags the cycle of economic growth by $\kappa$ periods, that is

$$
s_{2, t}=s_{1, t+\kappa}
$$

Note that positive values of $\kappa$ correspond to the situation that the cycle of $y_{2, t}$ leads the cycle of $y_{1, t}$ by $\kappa$ periods, whereas negative values correspond to a lag of $|\kappa|$ periods. We may treat the lead time $\kappa$ as an unknown parameter to be estimated.

As discussed in the introduction, stylized facts show that on average leading indicators have a longer lead time when entering a recession than when entering an expansion. To capture this phenomenon, we consider a new specification of the state vectors accompanying the MS-VAR model (3) such that $s_{2, t}$ leads $s_{1, t}$ by $\kappa_{1}$ periods at peaks and by $\kappa_{2}$ periods at troughs. This may be formalized by defining $s_{2, t}$ as

$$
s_{2, t}= \begin{cases}\prod_{i=\kappa_{1}}^{\kappa_{2}} s_{1, t+i} & \text { if } \kappa_{1} \leq \kappa_{2} \\ 1-\prod_{i=\kappa_{2}}^{\kappa_{1}}\left(1-s_{1, t+i}\right) & \text { if } \kappa_{1}>\kappa_{2} .\end{cases}
$$

To understand that this specification indeed gives rise to the desired asymmetric lead times, consider the case where $\kappa_{1} \leq \kappa_{2}$. Defining $s_{2, t}$ as the product from $s_{1, t+\kappa_{1}}$ to 
$s_{1, t+\kappa_{2}}$, essentially implies that recessions in $y_{2, t}$ start $\kappa_{1}$ periods before recessions in $y_{1, t}$, while they end $\kappa_{2}$ periods earlier. Note that, consequently, recessions in $y_{2, t}$ are $\left|\kappa_{2}-\kappa_{1}\right|$ periods shorter than recessions in $y_{1, t}$. On the other hand, if $\kappa_{1}>\kappa_{2}$, recessions in $y_{2, t}$ are $\left|\kappa_{1}-\kappa_{2}\right|$ periods longer than recessions in $y_{1, t}$. Obviously, lengthening of the recession periods is equivalent to shortening expansions. For that reason we define $s_{2, t}$ in $(8)$ in terms of the product over $\left(1-s_{1, t}\right)$ in this case.

Note that the specification of $s_{2, t}$ in (8) embeds the specifications with a synchronous common cycle $\left(\kappa_{1}=\kappa_{2}=0\right)$, and with a non-synchronous common cycle with symmetric lead/lag times at peaks and troughs $\left(\kappa_{1}=\kappa_{2}\right)$ as special cases. This facilitates testing for the degree of interrelation between the two cycles. The four specifications of the state vectors $s_{j, t}$, for $j=1,2$, discussed above are illustrated in Table 1.

\section{- Insert Table 1 about here -}

The bivariate MS-VAR(1) model in (3) may be extended in several directions to make it more realistic and useful in empirical practice. First, we may want to consider multiple coincident indicator variables, based on the original idea of Burns and Mitchell (1946, p.3) that the business "cycle consist of expansions (and recessions) occurring at about the same time in many economic activities." Similarly, it may be beneficial to include multiple leading indicator variables, as different recessions have different sources and characteristics and thus may be signaled by different leading indicators, see Stock and Watson (2003), among others. This may be accommodate by taking $y_{j, t}$ to be a $\left(m_{j} \times 1\right)$ vector, for $j=1,2$, such that the model includes $m_{1}$ coincident indicators and $m_{2}$ leading indicators. In case both $m_{1}>1$ and $m_{2}>1$, it may be cumbersome to clearly define the relationships between the states $s_{j, t}$, $j=1, \ldots, m_{1}+m_{2}$ directly as in (8), as now there are $m_{1} \times m_{2}$ different lead/lag times to consider. A possible solution then is to employ a dynamic factor structure as in Chauvet (1998), where all coincident and leading indicators are related with a certain lead/lag time to a latent common factor that exhibits regime-switching behavior.

Second, the model may be extended to incorporate higher-order dynamics in the coincident and leading indicators. For any lag order $k \geq 0$, the general MS-VAR 
model reads

$$
\begin{aligned}
\left(Y_{t}-\mathcal{M}_{\mathcal{S}_{t}}\right) & =\Phi_{1}\left(Y_{t-1}-\mathcal{M}_{\mathcal{S}_{t-1}}\right)+\cdots+\Phi_{k}\left(Y_{t-k}-\mathcal{M}_{\mathcal{S}_{t-k}}\right)+\mathcal{E}_{t} \\
& =\sum_{i=1}^{k} \Phi_{i}\left(Y_{t-i}-\mathcal{M}_{\mathcal{S}_{t-i}}\right)+\mathcal{E}_{t}
\end{aligned}
$$

or, using lag polynomial notation

$$
\Phi(L) Z_{t}=\left(I-\Phi_{1} L-\cdots-\Phi_{k} L^{k}\right) Z_{t}=\mathcal{E}_{t},
$$

where $Z_{t}=Y_{t}-\mathcal{M}_{\mathcal{S}_{t}}$.

A third possible extension of the model concerns the possibility of multiple regimes. Several applications of Markov switching models to US GDP, for example, have found that allowing for a third regime to capture the so-called 'bounce-back effect', that is a short period of rapid recovery following recessions, considerably improves the description of the cyclical dynamics of output, see Sichel (1994), Boldin (1996), and Clements and Krolzig (2003), among others. In the case of multiple regimes, specifying the lead/lag structure of the regime-switches for the different variables in the model may be complicated and has to be done with care.

Fourth, a model specification in which the transition probabilities of the Markov processes $s_{j, t}$, for $j=1,2$, depend on observed explanatory variables may be considered, see Diebold et al. (1994), Filardo (1994), and Diebold and Rudebusch (1996), among others.

Fifth and finally, regime-dependent heteroskedasticity and correlations among the shocks $\mathcal{E}_{t}$ may be captured by replacing the assumption $\mathcal{E}_{t} \sim N(0, \Sigma)$ in (3) by $\mathcal{E}_{t} \mid \mathcal{S}_{t} \sim N\left(0, \Sigma_{\mathcal{S}_{t}}\right)$. In the empirical application below we consider another type of dynamics in the error (co-)variances to accommodate the effects of the 'Great Moderation', that is the large and persistent decline in volatility of US macro-economic time series since the mid-1980s, see McConnell and Perez-Quiros (2000), Sensier and van Dijk (2004), and Herrera and Pesavento (2005), among others. Specifically, we allow for a single structural break in the covariance matrix of $\mathcal{E}_{t}$ :

$$
\begin{aligned}
\Sigma_{t} & = \begin{cases}\Omega_{0} & \text { if } t<\tau \\
\Omega_{1} & \text { if } t \geq \tau\end{cases} \\
& =\Omega_{0} \mathbb{I}[t<\tau]+\Omega_{1} \mathbb{I}[t \geq \tau],
\end{aligned}
$$


where $\mathbb{I}[\cdot]$ denotes the indicator function, taking the value one if the condition in brackets is true and zero otherwise, and $\Omega_{0}$ and $\Omega_{1}$ are $(2 \times 2)$ covariance matrices. We treat the break point $\tau$ as an unknown parameter to be estimated.

\section{$3 \quad$ Estimation and inference}

Parameter estimation and inference on the regimes in MS-(V)AR models is commonly done using maximum likelihood coupled with the EM-algorithm, see Hamilton $(1989,1994)$ for details. However, as we want to conduct inference on the discrete lead/lag time parameters $\kappa_{1}$ and $\kappa_{2}$ in (8), a frequentist approach is not feasible. We therefore adopt a Bayesian approach. In Section 3.1 we derive the likelihood function of the model. Sections 3.2 and 3.3 discuss the prior specification and posterior simulation.

\subsection{The likelihood function}

We first derive the complete data likelihood function. We focus on the derivation for the bivariate MS-VAR model (9) with asymmetric lead/lag structure as given in (8). The likelihood of the other specifications can be derived in a similar way.

Following Hamilton (1989) and Paap and van Dijk (2003) we replace $\mathcal{M}_{\mathcal{S}_{t}}$ by

$$
\mathcal{M}_{\mathcal{S}_{t}}=\Gamma_{0}+\Gamma_{1} \odot \mathcal{S}_{t}
$$

where $\odot$ denotes the Hadamard or element-by-element product and where $\mathcal{S}_{t}$ is given in (4) with (8). Hence, $\Gamma_{0}=\left(\mu_{1,0}, \mu_{2,0}\right)^{\prime}$ and $\Gamma_{1}=\left(\mu_{1,1}-\mu_{1,0}, \mu_{2,1}-\mu_{2,0}\right)^{\prime}$. Model (9) now reads

$$
\left(Y_{t}-\Gamma_{0}-\Gamma_{1} \odot \mathcal{S}_{t}\right)=\sum_{i=1}^{k} \Phi_{i}\left(Y_{t-i}-\Gamma_{0}-\Gamma_{1} \odot \mathcal{S}_{t-i}\right)+\mathcal{E}_{t}, \quad \mathcal{E}_{t} \sim N\left(0, \Sigma_{t}\right)
$$

for $t=k+1, \ldots, T$, where $T$ denotes the sample size and $\Sigma_{t}$ is specified in (11).

The conditional density of $Y_{t}$ for this model given the past and current states $\mathcal{S}^{t}=\left\{\mathcal{S}_{1}, \ldots, \mathcal{S}_{t}\right\}$ and given the past observations $Y^{t-1}=\left\{Y_{1}, \ldots, Y_{t-1}\right\}$ is given by

$$
f\left(Y_{t} \mid Y^{t-1}, \mathcal{S}^{t}, \Gamma_{0}, \Gamma_{1}, \Omega_{0}, \Omega_{1}, \Phi, \kappa_{1}, \kappa_{2}, \tau\right)=\frac{1}{(\sqrt{2 \pi})^{2}}\left|\Sigma_{t}\right|^{-\frac{1}{2}} \exp \left(-\frac{1}{2} \mathcal{E}_{t}^{\prime} \Sigma_{t}^{-1} \mathcal{E}_{t}\right)
$$


where $\mathcal{E}_{t}$ follows from (13). Hence the complete data likelihood function for model (13) conditional on the first $k$ observations $Y^{k}$ equals

$$
\begin{aligned}
\mathcal{L}\left(Y^{T}, \mathcal{S}^{T} \mid Y^{k}, \theta\right)=p^{\mathcal{N}_{0,0}}(1-p)^{\mathcal{N}_{0,1}} q^{\mathcal{N}_{1,1}}(1-q)^{\mathcal{N}_{1,0}} & \\
& \times \prod_{t=k+1}^{T} f\left(Y_{t} \mid Y^{t-1}, \mathcal{S}^{t}, \Gamma_{0}, \Gamma_{1}, \Omega_{0}, \Omega_{1}, \Phi, \kappa_{1}, \kappa_{2}, \tau\right),
\end{aligned}
$$

where $\theta=\left\{\Gamma_{0}, \Gamma_{1}, \Omega_{0}, \Omega_{1}, \Phi, \kappa_{1}, \kappa_{2}, \tau, p, q\right\}$, and where $\mathcal{N}_{i, j}$ denotes the number of transitions from state $i$ to state $j$. The unconditional likelihood function $\mathcal{L}\left(Y^{T} \mid Y^{k}, \theta\right)$ can be obtained by summing over all possible realizations of $\mathcal{S}^{T}$

$$
\mathcal{L}\left(Y^{T} \mid Y^{k}, \theta\right)=\sum_{s_{1}=0}^{1} \sum_{s_{2}=0}^{1} \cdots \sum_{s_{T}=0}^{1} \mathcal{L}\left(Y^{T}, \mathcal{S}^{T} \mid Y^{k}, \theta\right) .
$$

The complete data likelihood in case $\kappa=\kappa_{1}=\kappa_{2}$ follows directly from (15). In case we have separate cycles for the two series in $Y_{t}$ we have to extend (15) with the likelihood contribution of the second cycle in a straightforward manner.

\subsection{Prior specification}

We opt for a prior specification that is relatively uninformative compared to the information in the likelihood. For the transition probabilities $p$ and $q$, we take independent and uniformly distributed priors on the unit interval $(0,1)$

$$
p(p)=\mathbb{I}[0<p<1] \quad \text { and } \quad p(q)=\mathbb{I}[0<q<1]
$$

Under flat priors for $p$ and $q$ special attention must be paid to the priors for $\Gamma_{0}$ and $\Gamma_{1}$. It is easy to show that the likelihood has the same value if we switch the role of the states and change the values of $\Gamma_{0}, \Gamma_{1}, p$ and $q$ into $\Gamma_{0}+\Gamma_{1},-\Gamma_{1}, q$ and $p$ respectively, see Frühwirth-Schnatter (2001). This complicates proper posterior analysis if we are interested in the values of $\Gamma_{0}$ and $\Gamma_{1}$, see also Geweke (2007) for a discussion. Following Paap and van Dijk (2003) we take priors for $\Gamma_{0}$ and $\Gamma_{1}$ on subspaces which identify the regimes, that is,

$$
\begin{aligned}
p\left(\Gamma_{0}\right) & \propto \begin{cases}1 & \text { if } \Gamma_{0} \in\left\{\Gamma_{0} \in \mathbb{R}^{2} \mid \Gamma_{0,1}>0\right\} \\
0 & \text { elsewhere },\end{cases} \\
p\left(\Gamma_{1} \mid \Gamma_{0}\right) & \propto \begin{cases}1 & \text { if } \Gamma_{1} \in\left\{\Gamma_{1} \in \mathbb{R}^{2} \mid \Gamma_{0,1}+\Gamma_{1,1} \leq 0\right\} \\
0 & \text { elsewhere. }\end{cases}
\end{aligned}
$$


Hence, we impose that growth rate $\mu$ for the first series is positive if $s_{1, t}=0$ and negative if $s_{1, t}=1$. For the model specification with two independent cycles or, put differently, two independent Markov processes $s_{1, t}$ and $s_{2, t}$ we take the priors given in (17) for both sets of transition probabilities. In that case, the prior for $\Gamma_{0}$ and $\Gamma_{1}$ as given in (18) is augmented with the additional restrictions $\Gamma_{0,2}>0$ and $\Gamma_{0,2}+\Gamma_{1,2}$ for identification of the regimes of $y_{2, t}$.

For the shift parameters $\kappa_{j}$ we take a discrete uniform prior

$$
p\left(\kappa_{j}\right)= \begin{cases}\frac{1}{2 c_{j}+1} & \text { if } \kappa_{j} \in\left\{-c_{j}, \ldots, 0, \ldots, c_{j}\right\} \\ 0 & \text { elsewhere. }\end{cases}
$$

for $j=1,2$. Hence, we allow for a maximum lead/lag time of $c_{j}$ periods. The same prior is used for the lead time $\kappa$ in the model specification with a non-synchronous common cycle but equal lead times at peaks and troughs based on (7).

For the autoregressive parameters we use flat priors

$$
p\left(\Phi_{i}\right) \propto 1, \quad \text { for } i=1, \ldots, k-1 .
$$

and for $\Omega_{j}$ we take the uninformative prior

$$
p\left(\Omega_{j}\right) \propto\left|\Omega_{j}\right|^{-3 / 2}
$$

for $j=0,1$. This prior results from a standard Wishart prior by letting the degrees of freedom approaching zero, see Geisser (1965).

Finally, for the break parameter $\tau$ we take a discrete uniform prior

$$
p(\tau)= \begin{cases}\frac{1}{T-k-2 b} & \text { if } \tau \in\{k+b+1, \ldots, T-b\} \\ 0 & \text { elsewhere }\end{cases}
$$

hence not allowing for a break in the first and last $b$ observations of the sample period.

The joint prior for the model parameters $p(\theta)$ is given by the product of (17)$(22)$.

\subsection{Posterior distributions}

The posterior distribution for the model parameters of the Markov switching vector autoregressive model is proportional to the product of the prior $p(\theta)$ and the 
unconditional likelihood function $\mathcal{L}\left(Y^{T} \mid Y^{k}, \theta\right)$. To obtain posterior results we use the Gibbs sampling algorithm of Geman and Geman (1984) together with the data augmentation method of Tanner and Wong (1987). The unobserved state variables $\left\{\mathcal{S}_{t}\right\}_{t=1}^{T}$ are simulated alongside the model parameters $\theta$, see Albert and Chib (1993), McCulloch and Tsay (1994), Chib (1996) and Kim and Nelson (1999), among others.

The Gibbs sampler is an iterative algorithm, where one consecutively samples from the full conditional posterior distributions of the model parameters. This produces a Markov chain, which converges under mild conditions. The resulting draws can be considered as a sample from the posterior distribution, see Smith and Roberts (1993) and Tierney (1994) for details. In Appendix A we derive the full conditional posterior distributions resulting from $p(\theta) \mathcal{L}\left(Y^{T}, \mathcal{S}^{T} \mid Y^{k}, \theta\right)$ for the model specification with asymmetric lead/lag structure.

\section{Empirical results}

We apply the Markov switching VAR models proposed in Section 2 to examine the lead times at business cycle peaks and troughs of the Composite Leading Index (CLI) as issued by The Conference Board. As a proxy of economic growth we consider seasonally adjusted US Industrial Production (IP). IP is one of the four series that comprise The Conference Board's Composite Coincident Index. Both time series are transformed to monthly growth rates. The sample period runs from January 1959 to June 2004. The estimation results reported in this section use the revised data as available in July 2004. The next section considers out-of-sample forecasting of turning points and IP growth based on real-time data.

\section{- Insert Figure 1 about here -}

Figure 1 displays a time series plot of the log levels and monthly percentage growth rates of both series, together with the recession periods as determined by the National Bureau of Economic Research (NBER). In general, the leading index seems to have a similar cyclical pattern as the industrial production series, but with turning points cleary occurring earlier. In addition, the visual evidence in Figure 1 already suggests that the CLI turning points have a longer lead time for business cycle peaks than for troughs. We apply the four different specifications of the Markov switching 
model discussed in Section 2 to investigate more formally whether this indeed is the case, and to examine by how many periods the leading indicator actually is leading at peaks and troughs. In addition, for comparison we include a linear vector autoregressive model, which can be obtained from (9) by setting $\mathcal{M}_{\mathcal{S}_{t}}=\mathcal{M}$ for all $t$. In the first part of this section we discuss results from the models that do not allow for a structural break in volatility. In the second part we consider models that incorporate a single structural break in volatility as in (11).

\subsection{No structural break in volatility}

To perform inference we use the Bayesian approach as discussed in Section 3 with the prior specifications given in Section 3.2. We set the parameters $c_{1}$ and $c_{2}$ in the priors for the lead/lag times $\kappa_{1}$ and $\kappa_{2}$ equal to 12 , which implies that we allow for a maximum non-synchronicity of one year in the cycles of both series. We consider several specifications for the autoregressive dynamics in (9). Unreported Bayes factors based on moderately informative priors on $\Phi$ indicate that a lag order $k=1$ with additional restrictions $\phi_{1,1}=\phi_{2,1}=0$ is most appropriate, see Hamilton and Perez-Quiros (1996) for a similar specification. Hence, only lagged CLI growth enters the equations for both IP and CLI.

\section{- Insert Table 2 about here -}

Posterior results of the five estimated models are shown in Table 2, based on 100,000 simulations following a burn-in period of 10,000 iterations. The first panel of the table shows that in the linear VAR model the posterior mean of the average monthly growth rate over the sample period is $0.26 \%$ for industrial production and $0.12 \%$ for the composite leading index. In the MS-VAR models we observe clear differences in the average growth rates during with recession and expansion periods. Depending on the model specification, the posterior means of the average growth rates during expansions are between $0.39 \%$ and $0.46 \%$ for the IP series. For recessions the posterior means are between $-1.09 \%$ and $-0.84 \%$. The posterior mean of the probability of staying in an expansion regime is about 0.97 , while the probability of staying in a recession regime is considerably lower at about 0.82 . This obviously reflects the fact that recessions typically last much shorter than recessions. Based on these average transition probability estimates, the expected duration of a recession 
is between five and six months, compared to 33 months for expansions. Note that there is much more variability in the posterior means of the probability of staying in a recession than for the expansion staying probability across model specifications. In particular, the probability of staying in recession increases to 0.86 in the nonsynchronous common cycle specification of Hamilton and Perez-Quiros (1996).

The fourth panel of Table 2 shows that in the model with a non-synchronous common cycle (7) the posterior mean of the lead time of the CLI is just over four months. This corresponds with the lead time of one quarter as found in Hamilton and Perez-Quiros (1996), who used quarterly GNP and CLI data. In the novel model specification allowing for asymmetric lead/lag times $\kappa_{1}$ and $\kappa_{2}$, the posterior mean of the lead time at peaks is about 6.3 months compared to about 4.0 months at troughs. This confirms the informal visual evidence in Figure 1 that the CLI signals oncoming recessions earlier than expansions.

The bottom panel of the table shows the marginal likelihoods of the five models. As we have proper priors on the transitions probabilities we can compare the marginal likelihoods of the four Markov switching models to assess the appropriateness of the different cycle specifications. The marginal likelihood of the model with asymmetric lead/lag structure is clearly smaller than for the other models. The Bayes factor compared with the non-synchronous common cycle specification is $\exp (7.7) \approx 2208.4$ and hence, there is strong posterior evidence for the more general specification with different lead/lag times at peaks and troughs.

\section{- Insert Figure 2 about here -}

We proceed with judging the different model specifications on their ability to signal turning points and in particular their ability to identify recession periods. Figure 2 shows the posterior means of the state variables $s_{j, t}, j=1,2$ for the four Markov switching models. The shaded areas indicate recession periods defined as six consecutive months where the posterior mean of $s_{j, t}$ is larger than 0.5. This corresponds with the popular rule of thumb which says that the economy is in recession whenever economic growth is negative during two consecutive quarters. We compare our recession periods with those based on the NBER turning points, thus assuming that the latter are correct. We do note however that our analysis is solely based on cycles in IP, whereas the NBER focusses on multiple indicators, 
including real GDP, real income, employment, industrial production, and wholesaleretail sales. The bottom graph reveals that the model with an asymmetric lead/lag time at peaks and troughs as (8) indicates all the official NBER-dated recessions that occurred during the sample period. This includes the two most recent recessions in 1991 and 2001, both of which are missed by the other three specifications (except for the 2001 recessions in the non-synchronous common cycle specification with symmetric lead/lag times). In addition, the specifications with independent cycles and with a synchronous common cycle fail to identify the 1970 recession. All four models also fail to pick up the recession in 1980 in IP, but this may be attributed to the fact that this recession "officially" lasted for six months only, and hence is difficult to detect by construction. Note that the asymmetric lead/lag model specification does indicate a recession period for the CLI and a short period with negative growth in IP in 1980. Finally, the asymmetric lead/lag model indicates an additional recession period around 1967, which corresponds with a growth rate cycle recession. ${ }^{1}$

\subsection{Structural break in volatility}

Next, we consider the same set of models but allowing for a single structural break in the covariance matrix as in (11). The parameter $b$ in the prior for the break date is set equal to 6 , so that we do not allow for a break in the (co-)variances in the first and last six observations. The other prior specifications are kept the same. Posterior results for the five models are shown in Table 3.

\section{- Insert Table 3 about here -}

The bottom panel of the table shows that the marginal likelihoods of the models with a structural break are clearly smaller than for the models without, and hence there is strong posterior evidence for a structural break in volatility. The posterior mode of the break point parameter $\tau$ is 1984.02 in all model specifications, which corresponds with the break point estimate for GDP volatility as reported by McConnell and Perez-Quiros (2000), among others. Figure 3 displays the posterior density of the break parameter for the model with an asymmetric lead/lag structure

\footnotetext{
${ }^{1}$ According to the Economic Cycle Research Institute, see http://www. businesscycle.com.
} 
for the period January 1982-December 1986. Almost all posterior mass is located in the years 1983 and 1984. In the MS-VAR model with asymmetric lead/lag times, the posterior means of the variances are such that for IP the variance after the break is about $30 \%$ of the variance before. For the leading indicator the reduction in variance also is very large at $64 \%$. Computing the posterior means of the correlation between the IP and CLI growth rates, we obtain 0.458 (0.052) and 0.291 (0.066) before and after the break, respectively, where the posterior standard deviations are in parentheses. This suggests that the strength of the co-movement between the series also declined.

\section{- Insert Figure 3 about here -}

The first panel of Table 3 shows that the posterior means of average growth rates over the observation period become somewhat smaller if we allow for a structural break, at $0.25 \%$ and $0.11 \%$ for IP and the CLI, respectively. This also holds for the posterior means of the growth rates in the expansion and recession periods in the different Markov switching models.

The posterior mean of the lead time of the leading indicator in the non-synchronous common cycle model in (7) now is about 3.6 months, slightly more than half a month less than for the model without the volatility break. The posterior means of $\kappa_{1}$ and $\kappa_{2}$ in the model with asymmetric lead/lag times as in also change slightly, but such that their difference becomes even larger with the posterior mean lead time at peaks being 7.3 months compared to 3.5 months at troughs. Figure 4 displays the posterior distribution of the $\kappa_{j}$ parameters, for $j=1,2$ in this model specification, showing that the posterior mode is $\kappa_{1}=6$ and $\kappa_{2}=4$. The posterior probability that $\kappa_{1}=\kappa_{2}$ is only 0.05 , providing complementary evidence that the lead times at the start of recessions and expansions really are different.

\section{- Insert Figure 4 about here -}

Again we find that the marginal likelihood of the model with an asymmetric lead/lag structure is higher than for the other cycle specifications, although the differences in the log marginal likelihood value are smaller than for the models without a volatility break. The Bayes factor compared with the non-synchronous common 
cycle specification is $\exp (2.2) \approx 9.0$ and hence, there is still considerable posterior evidence for the asymmetric lead/lag specification.

\section{- Insert Figure 5 about here -}

Figure 5 shows the posterior means of the state variables $s_{j, t}$, for $j=1,2$. The most notable difference with the corresponding graphs in Figure 2, is that the alternative, simpler cycle specifications are able to identify the 1991 and 2001 recessions when a break in volatility is allowed for, see panels (b)-(d). Note, however, that the model with a non-synchronous common cycle with an asymmetric lead/lag structure is more timely, in the sense that it signals these recessions (by showing an increase in the probability that $s_{2, t}=1$ ) quite a bit earlier than the other specifications. In fact, this occurs for all other recessions during the sample period, again demonstrating the advantage of allowing for different lead times at peaks and troughs. Finally, even when a volatility break is allowed, only the asymmetric model specification is capable of identifying the short recession in 1980.

\section{$5 \quad$ Real-time business cycle dating and forecasting}

The full-sample estimation results discussed in the previous section demonstrate that using the CLI within an MS-VAR model delivers an accurate description of US business cycle dynamics ex post. The practical usefulness of leading indicator variables, however, crucially hinges upon their ability to signal changes in the business cycle ex ante. Furthermore, as both the CLI and IP are subject to substantial revisions after their initial release, a realistic assessment of this issue requires the use of real-time data that was actually available when the forecasts were supposed to be made. Two related aspects of real-time performance are of interest. First, we consider real-time business cycle dating, as in Chauvet and Piger (2007), and examine how quickly the different models provide a reliable signal that the business cycle regime has changed. Second, we consider genuine out-of-sample forecasting of both turning points and output growth in real time. A number of previous studies examining the real-time predictive ability of the CLI have rendered mixed results, depending on the choice of time series model as well as the coincident indicator variable(s), see Diebold and Rudebusch (1991), Hamilton and Perez-Quiros (1996), Camacho and Perez-Quiros 
(2002), Filardo (1999, 2004), and McGuckin et al. (2007), among others. Here we examine whether there is any added value of allowing for different lead times at peaks and troughs for predictive accuracy.

Our real-time data set for the CLI and IP consists of 200 releases, or vintages, from October 1987 until July 2004. Each vintage contains a complete time series of monthly observations from January 1959 until one month prior to the release date. Except for McGuckin et al. (2007), previous studies examining the real-time predictive ability of the CLI use revised data as available at present for the coincident indicator(s) or output measure, based on the idea that the revised data is closer to the truth that we (should) aim to forecast. However, this comes at the cost of making the real-time experiment less realistic as revisions in output measures are substantial, see Swanson and van Dijk (2006) for a recent assessment for monthly IP. As we would like to approximate the actual possibilities of a business cycle analyst as closely as possible, we make use of real-time IP data instead.

We construct real-time estimates and forecasts of the business cycle indicator $s_{1, t}$ and the monthly IP growth rate $y_{1, t}$ for each vintage in the period October 1987-July 2004, as follows. Using the data release of month $T$, which contains observations until month $T-1$, we first obtain the posterior distribution of the model parameters. Of particular interest is the posterior distribution of the state variable $p\left(s_{1, t} \mid Y^{T-1}\right)$ for $t=1,2, \ldots, T-1$, as this can be used for real-time dating of business cycle peaks and troughs. Next, we determine the predictive densities $p\left(s_{1, T-1+h} \mid Y^{T-1}\right)$ and $p\left(y_{1, T-1+h} \mid Y^{T-1}\right)$ for $h=1,2, \ldots$ Draws from these predictive densities can easily be obtained from the Gibbs output for the posterior distribution. Given a draw from the posterior of the parameters and states $\mathcal{S}_{1}, \ldots, \mathcal{S}_{T-1}$, we simply simulate future observations taking the model as data generating process. We use the means of the predictive distributions as point forecasts. This implies that the forecast for $s_{1, T-1+h}$ is the predictive probability that $s_{1, T-1+h}$ equals 1 or, put differently, the probability that month $T-1+h$ is part of a recession. We consider forecast horizons up to $h=12$ steps ahead, where it should be noted that in fact the one-step ahead prediction is a nowcast as it is made at the end of month $T$. 


\subsection{Business cycle dating results}

For a business cycle dating procedure to be useful in real time, it should strike a balance between the speed at which regime shifts are detected and the accuracy of estimated turning point dates. The posterior distribution $p\left(s_{1, t} \mid Y^{T-1}\right)$ constructed at the end of month $T$ delivers smoothed probability estimates $\operatorname{Pr}\left[s_{1, t}=1 \mid Y^{T-1}\right]$ for $t=1,2, \ldots, T-1$. To convert these recession probabilities into turning point estimates, we may again use a specific dating rule as we did in Section 4, where we defined a recession as a period of six consecutive months where the recession probability exceeds 0.5. Some business cycle analysts, however, may be more inclined to accept also weaker signals, if the speed of detection is of utmost importance. Others may prefer to wait longer, in order to gain accuracy and certainty about the dates obtained. For this reason, instead, we visualize the recession probabilities and leave the exact dating rule to the reader.

\section{- Insert Figures 6 and 7 about here -}

Figures 6 and 7 show the real-time recession probabilities obtained using data vintages before, during and after the recessions in 1990-1991 and 2001, respectively. Each row in these graphs gives recession probabilities based on the vintage released at the end of the month as marked on the vertical axis, where the in-sample estimates and out-of-sample predictions are separated by the stepwise diagonal line. Looking across columns for a specific row thus shows the business cycle dating for a particular vintage. If the probability estimates change such that the color of the graph turns from blue to red at a certain calendar month and remain red consistently thereafter, we gain confidence that this date should be marked as a business cycle peak. A change from red to blue similarly indicates a trough. Conversely, looking across rows reveals how the assessment of a particular calendar month changes across data releases when focusing on a specific column and, more generally, how the business cycle dating changes across data vintages.

For the July 1990 - March 1991 recession, we find that the models with the synchronous common cycle and the non-synchronous common cycle with identical lead times at peaks and troughs are clearly outperformed by the models with independent cycles and an asymmetric non-synchronous common cycle, as the signals of 
the first two models are not particularly strong and, perhaps more importantly, not persistent. Comparing the latter two models, the non-synchronous common cycle specification with different lead times at peaks and troughs provides stronger signals in the sense that the estimated probabilities are closer to zero or one. Also, this model does not provide false signals around February 1991 and 1992, which are observed with the independent cycles specification. As regards the speed of detection of the business cycle peak, we observe a first string of recession probabilities higher than 0.5 for the data release of January 1991. The probabilities color red persistently as of April, which is around the same date as the NBER's announcement of the peak. However, as early as July 1991 our model indicates that the recession had ended in April. The NBER announced the end of this recession in December 1992, nearly one and a half year after the model's time of detection. Concerning the accuracy of our procedure, we note a discrepancy of four months for the peak and one month for the trough.

Looking at the results for the March - November 2001 recession shown in Figure 7, the two non-synchronous common cycle models provide much more clear signals than the independent cycles and synchronous common cycle specifications. In a one-to-one comparison of the first two models, again the asymmetric specification is to be preferred, as it provides sharper regime switches. Also it does not show the false signals at the end of 2002. Based on the symmetric model we would have concluded that the recession started seven months earlier than it started according to the NBER. Most probably as a result of the asymmetric lead/lag structure, our novel specification dates the beginning of the recession more sharply. As regards the speed of detection, we observe that the recession probabilities turn red for the vintage of July 2001, four months before the NBER's announcement of this peak. We detect the trough in May 2002, where NBER announced this trough in July 2003.

\section{- insert Figure 7 about here -}

\section{$5.2 \quad$ Forecasting results}

We conclude our analysis by evaluating the (relative) accuracy of real-time $h$-month ahead forecasts of the business cycle regime and of IP growth, for $h=1,2, \ldots, 12$. 
To evaluate the latter forecasts we use the mean squared forecast error (MSFE)

$$
\operatorname{MSFE}(h)=\frac{1}{T_{2}-h-T_{1}} \sum_{t=T_{1}-1}^{T_{2}-h}\left(y_{1, t+h}-\hat{y}_{1, t \mid t+h}\right)^{2}
$$

where $T_{1}$ and $T_{2}$ correspond to the first and last data vintages (October 1987 and July 2004), where $\hat{y}_{1, t \mid t+h}$ denotes the $h$-step ahead forecast made at time $t+1$ and where $y_{t+h}$ is the monthly IP growth rate resulting from the release of the series in July 2004. For the regime variable $s_{1, t}$ we use the turning point forecast error (TPFE)

$$
\operatorname{TPFE}(h)=\frac{1}{T_{2}-h-T_{1}} \sum_{t=T_{1}-1}^{T_{2}-h}\left(\mathrm{NBER}_{t+h}-\hat{s}_{1, t \mid t+h}\right)^{2}, \quad \text { for } h=1, \ldots, 12,
$$

where $\hat{s}_{1, t \mid t+h}$ denotes the $h$-step ahead forecast of the state variable made at time $t+1$, and where $\mathrm{NBER}_{t+h}$ is a binary variable which equals 1 if, according to the NBER turning points, the economy is in recession at time $t+h$.

To facilitate the forecast comparison, we take the most general model specification, that is, the asymmetric non-synchronous common cycle model as reference point. The first panel of Figure 8 displays ratios of the $\operatorname{TPFE}(h)$ for $h$-step ahead forecasts of probability of recession obtained from the MS-VAR models with independent cycles, with a synchronous common cycle and with a symmetric nonsynchronous common cycle relative to the asymmetric non-synchronous common cycle model. We observe that the asymmetric model outperforms the other models in forecasting turning points as all ratios exceed unity, except for the synchronous common cycle model at $h=12$ months ahead. The improvement in forecast accuracy is especially large for short horizons, in particular for the symmetric nonsynchronous common cycle specification of Hamilton and Perez-Quiros (1996). For horizons longer than 10 months the differences are relatively small. The model specification with a synchronous common cycle is second best for all horizons.

\section{- insert Figure 8 about here -}

We test whether the differences in TPFE's are statistically significant by means of the Diebold and Mariano (1995) test of equal predictive accuracy. The results in the first panel of Table 4 for forecast horizons $h=1,2,3,6,9$, and 12 months lead to 
slightly different conclusions than the graphical evidence in Figure 8. In particular, the asymmetric non-synchronous common cycle model provides significantly more accurate forecast than the symmetric variant only for one month ahead, despite the still sizable values of the TPFE ratios for longer horizons. The synchronous variant, although being second best is still significantly worse for forecast horizons of two and three months.

\section{- insert Table 4 about here -}

The second panel of Figure 8 displays the ratios of the $\operatorname{MSFE}(h)$ for $h=1, \ldots, 12$ for IP growth forecasts for the same three models relative to the asymmetric nonsynchronous cycle model. Differences in forecast performance are smaller but still the novel cycle specification outperforms the other models. The second panel of Table 4 displays the corresponding Diebold and Mariano (1995) test results. Comparing the general model with the variants with independent cycles and with a synchronous common cycle, the MSFE differences are significant for 1, 9 and 12 months ahead. The differences with the symmetric non-synchronous common cycle model are not statistically significant, except perhaps for the three months ahead forecasts.

In sum, our model produces sharper and more accurate turning point estimates, in particular for business cycle peaks. Concerning the speed of detection, our model proves to be advantageous especially to detect business cycle troughs. For the last two recessions, the troughs where detected over a year ahead of the NBER's announcements. The asymmetric non-synchronous common cycle model also provides more accurate forecasts than the other models, especially for turning points.

\section{Conclusions}

In this paper, we have developed a formal statistical approach to investigate whether the lead time of leading indicator variables is different at business cycle peaks and troughs. A novel Markov switching vector autoregressive model, where economic growth and leading indicators share a common Markov process determining the state but with different lead times at switches between the different regimes, was proposed for this purpose. The empirical application involving monthly industrial production and The Conference Board's CLI demonstrates the usefulness of the new 
model specification. For the period 1959-2004, we found that on average the CLI led IP by seven months at peaks, but only by three and a half months at troughs. Therefore, in terms of timeliness, the CLI is most useful for signalling oncoming recessions. In addition, in a real-time business cycle dating and forecasting exercise for the period from October 1987 to July 2004, we found that allowing for asymmetric lead times leads to more timely and precise identification of peaks and troughs for the 1990-1991 and 2001 recessions, as well as more accurate out-of-sample forecasts of turning points as well as IP growth rates. 


\section{A Full conditional posterior distributions}

\section{A.1 Sampling of $\Gamma_{0}$}

To sample $\Gamma_{0}$ we rewrite (13) as

$\Sigma_{t}^{-\frac{1}{2}}\left(\left(Y_{t}-\Gamma_{1} \odot \mathcal{S}_{t}\right)-\sum_{i=1}^{k} \Phi_{i}\left(Y_{t-i}-\Gamma_{1} \odot \mathcal{S}_{t-i}\right)\right)=\Sigma_{t}^{-\frac{1}{2}}\left(I_{m}-\sum_{i=1}^{k} \Phi_{i}\right) \Gamma_{0}+\Sigma_{t}^{-\frac{1}{2}} \mathcal{E}_{t}$,

where $I_{m}$ denotes the $(m \times m)$ identity matrix. This is a regression model with parameter $\Gamma_{0}$ and an error term with unit variance. Hence, the full conditional posterior distribution of $\Gamma_{0}$ is normal with mean $\left(X^{\prime} X\right)^{-1} X^{\prime} Z$ and covariance matrix $\left(X^{\prime} X\right)^{-1}$ where $Z=\left(Z_{k+1}^{\prime}, \ldots, Z_{T}^{\prime}\right)^{\prime}$ with $Z_{t}=\Sigma_{t}^{-\frac{1}{2}}\left(\left(Y_{t}-\Gamma_{1} \odot \mathcal{S}_{t}\right)-\sum_{i=1}^{k} \Phi_{i}\left(Y_{t-i}-\Gamma_{1} \odot \mathcal{S}_{t-i}\right)\right)$

and $X=\left(X_{k+1}^{\prime}, \ldots, X_{T}^{\prime}\right)^{\prime}$ with $X_{t}=\Sigma_{t}^{-\frac{1}{2}}\left(I_{m}-\sum_{i=1}^{k} \Phi_{i}\right)$, see, for example, Zellner (1971, Chapter III). The prior restriction for identification can easily be incorporated by sampling from truncated normal distributions.

\section{A.2 Sampling of $\Gamma_{1}$}

To sample $\Gamma_{1}$ we rewrite $(13)$ as

$\Sigma_{t}^{-\frac{1}{2}}\left(\left(Y_{t}-\Gamma_{0}\right)-\sum_{i=1}^{k} \Phi_{i}\left(Y_{t-i}-\Gamma_{0}\right)\right)=\Sigma_{t}^{-\frac{1}{2}}\left(I_{m} \odot\left(\mathcal{S}_{t}-\sum_{i=1}^{k} \Phi_{i} \mathcal{S}_{t-i}\right)\right) \Gamma_{1}+\Sigma_{t}^{-\frac{1}{2}} \mathcal{E}_{t}$

This is again a regression model with parameter $\Gamma_{1}$ and an error term with unit variance. The full conditional posterior distribution of $\Gamma_{1}$ is normal with mean $\left(X^{\prime} X\right)^{-1} X^{\prime} Z$ and covariance matrix $\left(X^{\prime} X\right)^{-1}$ where $Z=\left(Z_{k+1}^{\prime}, \ldots, Z_{T}^{\prime}\right)^{\prime}$ with $Z_{t}=$ $\Sigma_{t}^{-\frac{1}{2}}\left(\left(Y_{t}-\Gamma_{0}\right)-\sum_{i=1}^{k} \Phi_{i}\left(Y_{t-i}-\Gamma_{0}\right)\right)$ and $X=\left(X_{k+1}^{\prime}, \ldots, X_{T}^{\prime}\right)^{\prime}$ with $X_{t}=\Sigma_{t}^{-\frac{1}{2}} \times$ $\left(I_{m} \odot\left(\mathcal{S}_{t}-\sum_{i=1}^{k} \Phi_{i} \mathcal{S}_{t-i}\right)\right)$. Again, the prior restriction for identification can easily be incorporated by sampling from truncated normal distributions.

\section{A.3 Sampling of $\Phi$}

To sample $\Phi$ we note that (13) is a multivariate regression model with regression parameters $\Phi_{i}$ for $i=1, \ldots, k$. Define $Z_{t}=\left(Y_{t}-\Gamma_{0}-\Gamma_{1} \odot \mathcal{S}_{t}\right)$ and $Z^{j}=$ $\left(Z_{k-j+1}, \ldots, Z_{T-j}\right)^{\prime}$. This multivariate regression model can be written as

$$
Z=X \Phi+e
$$


where $Z=D Z^{0}, X=\left(D Z^{1}, D Z^{2}, \ldots, D Z^{k}\right), D=\operatorname{diag}\left(\Sigma_{k+1}^{-\frac{1}{2}}, \ldots, \Sigma_{T}^{-\frac{1}{2}}\right), \Phi=$ $\left(\Phi_{1}, \ldots, \Phi_{k}\right)^{\prime}$ and $e=\left(\Sigma_{k+1}^{-\frac{1}{2}} \mathcal{E}_{k+1}, \ldots, \Sigma_{T}^{-\frac{1}{2}} \mathcal{E}_{T}\right)^{\prime}$. Hence, the full conditional posterior distribution of $\Phi$ is a matric variate normal distribution with mean $\left(X^{\prime} X\right)^{-1} X^{\prime} Z$ and covariance matrix $I_{m} \otimes\left(X^{\prime} X\right)^{-1}$, see Zellner (1971, Chapter VIII). To sample in case we have zero restrictions on the elements of $\Phi$ we rewrite (A.3) in a univariate linear regression model with regression parameter vec $(\Phi)$ using the vec operator, that is,

$$
\operatorname{vec}(Z)=\operatorname{vec}(I \otimes X) \operatorname{vec}(\Phi)+\operatorname{vec}(e)
$$

and hence one can sample the nonzero elements of $\operatorname{vec}(\Phi)$ from a normal distribution with mean $\left(\tilde{X}^{\prime} \tilde{X}\right)^{-1}\left(\tilde{X}^{\prime} \operatorname{vec}(Z)\right)$ and covariance matrix $\left(\tilde{X}^{\prime} \tilde{X}\right)^{-1}$ where $\tilde{X}$ contains the columns of $\operatorname{vec}(I \otimes X)$ corresponding to the nonzero elements of $\operatorname{vec}(\Phi)$.

\section{A.4 Sampling of $\Omega_{0}$ and $\Omega_{1}$}

It is easy to see from the conditional likelihood (15) and the prior specification (21) that the full conditional posterior of density $\Omega_{0}$ and $\Omega_{1}$ is proportional to

$$
\begin{aligned}
p\left(\Omega_{0}, \Omega_{1} \mid s^{T}, \theta \backslash\left\{\Omega_{0}, \Omega_{1}\right\}, Y^{T}\right) & \propto\left|\Omega_{0}\right|^{-\frac{1}{2}(\tau-k)}\left|\Omega_{1}\right|^{-\frac{1}{2}(T-\tau+2)} \\
& \times \exp \left(-\frac{1}{2} \operatorname{tr}\left(\Omega_{0}^{-1}\left(\sum_{t=k+1}^{\tau-1} \mathcal{E}_{t} \mathcal{E}_{t}^{\prime}\right)+\Omega_{1}^{-1}\left(\sum_{t=\tau}^{T} \mathcal{E}_{t} \mathcal{E}_{t}^{\prime}\right)\right)\right)
\end{aligned}
$$

and hence the covariance matrices $\Omega_{0}$ and $\Omega_{1}$ can be sampled from inverted Wishart distributions with scale parameters $\left(\sum_{t=k+1}^{\tau-1} \mathcal{E}_{t} \mathcal{E}_{t}^{\prime}\right)$ and $\left(\sum_{t=\tau}^{T} \mathcal{E}_{t} \mathcal{E}_{t}^{\prime}\right)$ and degrees of freedom $\tau-k-1$ and $T-\tau+1$, respectively, see Zellner (1971, p. 395).

\section{A.5 Sampling of $p$ and $q$}

From the conditional likelihood function (15) it follows that the full conditional posterior densities of the transition parameters are given by

$$
\begin{aligned}
& p\left(p \mid s^{T}, \theta \backslash\{p\}, Y^{T}\right) \propto p^{\mathcal{N}_{0,0}}(1-p)^{\mathcal{N}_{0,1}} \\
& p\left(q \mid s^{T}, \theta \backslash\{q\}, Y^{T}\right) \propto q^{\mathcal{N}_{1,1}}(1-q)^{\mathcal{N}_{1,0}},
\end{aligned}
$$

where $\mathcal{N}_{i, j}$ again denotes the number of transitions from state $i$ to state $j$. This implies that the transition probabilities can be sampled from Beta distributions with parameters $\mathcal{N}_{0,0}+1 \& \mathcal{N}_{0,1}+1$, and $\mathcal{N}_{1,1}+1 \& \mathcal{N}_{1,0}+1$, respectively. In case 
we have separate state variables $s_{t}$ for the two series we can sample both transition probabilities separately.

\section{A.6 Sampling of $\tau$}

The full conditional posterior density of $\tau$ is given by

$$
\begin{aligned}
p\left(\tau \mid s^{T}, \theta \backslash\{\tau\}, Y^{T}\right) & \propto \mathbb{I}[b+k+1<\tau \leq T-b] \times\left|\Omega_{0}\right|^{-\frac{1}{2}(\tau-k)}\left|\Omega_{1}\right|^{-\frac{1}{2}(T-\tau+2)} \\
& \times \exp \left(-\frac{1}{2} \operatorname{tr}\left(\Omega_{0}^{-1}\left(\sum_{t=k+1}^{\tau-1} \mathcal{E}_{t} \mathcal{E}_{t}^{\prime}\right)+\Omega_{1}^{-1}\left(\sum_{t=\tau}^{T} \mathcal{E}_{t} \mathcal{E}_{t}^{\prime}\right)\right)\right)
\end{aligned}
$$

As $\tau$ has can only take discrete values on the range $(\nu+k+1, T-\nu]$ we can easily sample from its full conditional posterior distribution.

\section{A.7 Sampling of $\kappa$}

If our model contains non-synchronous cycles in both series we have to sample one or two $\kappa$ parameters. As the $\kappa$ parameters are discrete we can compute the value of the posterior distribution for $\kappa_{j} \in\left\{-c_{j}, \ldots, c_{j}\right\}$ and scale these values such that they add up to one. We can now easily sample a value for $\kappa$. Note that we can sample $\kappa_{1}$ and $\kappa_{2}$ at once from their joint full conditional distribution.

\section{A.8 Sampling of the states}

To sample the states, we need the full conditional posterior density of $s_{t}$, denoted by $p\left(s_{t} \mid s^{-t}, \theta, Y^{T}\right), t=1, \ldots, T$, where $s^{-t}=s^{T} \backslash\left\{s_{t}\right\}$. Since $s_{t}$ follows a first-order Markov process, it is easily seen that

$$
p\left(s_{t} \mid s^{-t}\right) \propto p\left(s_{t} \mid s_{t-1}\right) p\left(s_{t+1} \mid s_{t}\right)
$$

due to the Markov property. Hence, the full conditional distribution of $s_{t}$ is given by

$$
p\left(s_{t} \mid s^{-t}, \theta, Y^{T}\right) \propto p\left(s_{t} \mid s_{t-1}, \theta\right) p\left(s_{t+1} \mid s_{t}, \theta\right) \prod_{i=t-\kappa_{\min }}^{t+k+\kappa_{\max }} f\left(Y_{i} \mid Y^{i-1}, \mathcal{S}^{i}, \theta\right),
$$

where $f\left(Y_{t} \mid Y^{t-1}, \mathcal{S}^{t}, \theta\right)$ is defined in $(14), \kappa_{\max }=\max \left(\kappa_{1}, \kappa_{2}\right), \kappa_{\min }=\min \left(\kappa_{1}, \kappa_{2}\right)$ and the constant of proportionality can be obtained by summing over the two possible 
values of $s_{t}$. At time $t=T$ the term $p\left(s_{T+1} \mid s_{T}, \theta\right)$ drops out. The first $k$ states can be sampled from the full conditional distribution

$$
p\left(s_{t} \mid s^{-t}, \theta, Y^{T}\right) \propto p\left(s_{t} \mid s_{t-1}, \theta\right) p\left(s_{t+1} \mid s_{t}, \theta\right) \prod_{i=k+1}^{t+k+\kappa_{\max }} f\left(Y_{i} \mid Y^{i-1}, \mathcal{S}^{i}, \theta\right),
$$

for $t=1, \ldots, k$, where at time $t=1$ the term $p\left(s_{t} \mid s_{t-1}, \theta\right)$ is replaced by the unconditional density $p\left(s_{1} \mid \theta\right)$, which is a binomial density with probability $(1-p) /(2-p-q)$.

Sampling of the state variables can be done as follows. Take the most recent value of $s^{T}$ and sample the states backward in time, one after another, starting with $s_{T}$. After each step, the $t$-th element of $s^{T}$ is replaced by its most recent draw. 


\section{References}

Albert, J. H. and S. Chib (1993), Bayes Inference via Gibbs Sampling of Autoregressive Time Series subject to Markov Mean and Variance Shifts, Journal of Business Es Economic Statistics, 11, 1-15.

Boldin, M. D. (1996), A Check on the Robustness of Hamilton's Markov Switching Approach to the Economic Analysis of Business Cycles, Studies in Nonlinear Dynamics and Econometrics, 1, 35-46.

Burns, A. F. and W. C. Mitchell (1946), Measuring Business Cycles, National Bureau of Economic Research, New York.

Camacho, M. and G. Perez-Quiros (2002), This is What the Leading Indicators Lead, Journal of Applied Econometrics, 17, 61-80.

Chauvet, M. and J. D. Hamilton (2006), Dating Business Cycle Turning Points, in C. Milas, P. Rothman, and D. van Dijk (eds.), Nonlinear Time Series Analysis of Business Cycles, Elsevier, North-Holland, pp. 1-54.

Chauvet, M. and J. Piger (2007), A Comparison of the Real-Time Performance of Business Cycle Dating Methods, Journal of Business $\&$ Economic Statistics, to appear.

Chauvet, M. (1998), An Econometric Characterization of Business Cycle Dynamics with Factor Structure and Regime Switching, International Economic Review, 39, 969-996.

Chib, S. (1996), Calculating Posterior Distributions and Model Estimates in Markov Mixture Models, Journal of Econometrics, 75, 79-97.

Clements, M. P. and H.-M. Krolzig (2003), Business Cycle Asymmetries: Characterization and Testing Based on Markov-Switching Autoregressions, Journal of Business 83 Economic Statistics, 21, 196-211.

Diebold, F. X., J. Lee, and G. C. Weinbach (1994), Regime Switching and Endogenous Transition Probablities, in C. Hargreaves (ed.), Nonstationary Time Series 
Analysis and Cointegration, chap. 10, Oxford University Press, Oxford, pp. 283302.

Diebold, F. X. and R. S. Mariano (1995), Comparing Predictive Accuracy, Journal of Business \& Economic Statistics, 13, 253-263.

Diebold, F. X. and G. D. Rudebusch (1991), Forecasting Output with the Composite Leading Index: An Ex Ante Analysis, Journal of the American Statistical Association, 86, 603-610.

Diebold, F. X. and G. D. Rudebusch (1996), Measuring Business Cycles: A Modern Perspective, Review of Economics and Statistics, 78, 67-77.

Filardo, A. J. (1994), Business-Cycle Phases and Their Transitional Dynamics, Journal of Business \& Economic Statistics, 12, 299-308.

Filardo, A. J. (1999), How Reliable Are Recession Prediction Models?, Federal Reserve Bank of Kansas City Economic Review, 84, 35-55.

Filardo, A. J. (2004), The 2001 US Recession: What Did Recession Prediction Models Tell Us?, bank of International Settlements Working Paper No. 148.

Frühwirth-Schnatter, S. (2001), Markov Chain Monte Carlo Estimation of Classical and Dynamic Switching and Mixture Models, Journal of American Statistical Association, 96, 194-209.

Geisser, S. (1965), A Bayes Approach for Combining Correlated Estimates, Journal of the American Statistical Association, 60, 602-607.

Geman, S. and D. Geman (1984), Stochastic Relaxations, Gibbs Distributions, and the Bayesian Restoration of Images, IEEE Transaction on Pattern Analysis and Machine Intelligence, 6, 721-741.

Geweke, J. F. (2007), Interpretation and Inference in Mixture Models: Simple MCMC Works, Computational Statistics and Data Analysis, to appear.

Hamilton, J. D. and G. Perez-Quiros (1996), What Do the Leading Indicators Lead, Journal of Business, 69, 27-49. 
Hamilton, J. D. (1989), A New Approach to the Econometric Analysis of Nonstationary Time Series and Business Cycles, Econometrica, 57, 357-384.

Hamilton, J. D. (1994), Time Series Analysis, Princeton University Press, New Jersey.

Herrera, A.-M. and E. Pesavento (2005), The Decline in U.S. Output Volatility: Structural Changes and Inventory Investment, Journal of Business $\& 3$ Economic Statistics, 23, 462-472.

Kim, C.-J. and C. R. Nelson (1999), State-Space Models with Regime Switching: Classical and Gibbs-Sampling Approaches with Applications, MIT Press, Cambridge, MA.

Krolzig, H.-M. (1997), Markov Switching Vector Autoregressions: Modelling, Statistical Inference, and an Application to Business Cycle Inference, Springer, Berlin.

Marcellino, M. (2006), Leading Indicators: What Have We Learned?, in G. Elliott, C. W. Granger, and A. Timmermann (eds.), Handbook of Economic Forecasting, Elsevier, North-Holland, pp. 879-960.

McConnell, M. and G. Perez-Quiros (2000), Output Fluctuations in the United States: What Has Changed since the 1980s?, American Economic Review, 90, $1464-1476$.

McCulloch, R. E. and R. S. Tsay (1994), Statistical Analysis of Economic Time Series via Markov Switching Models, Journal of Time Series Analysis, 15, 523540.

McGuckin, R. H., A. Ozyildirim, and V. Zarnowitz (2007), A More Timely and Useful Index of Leading Indicators, Journal of Business 83 Economic Statistics, $25,110-120$.

Mitchell, W. C. and A. F. Burns (1938), Statistical Indicators of Cyclical Revivals, National Bureau of Economic Research, New York.

Paap, R. and H. K. van Dijk (2003), Bayes Estimates of Markov Trends in Possibly Cointegrated Series: An Application to US Consumption and Income, Journal of Business 86 Economic Statistics, 21, 547-563. 
Sensier, M. and D. van Dijk (2004), Testing for Volatility Changes in US Macroeconomic Time Series, Review of Economics and Statistics, 86, 833-839.

Sichel, D. E. (1994), Inventories and the Three Phases of the Business Cycle, Journal of Business \& Economic Statistics, 12, 269-277.

Smith, A. F. M. and G. O. Roberts (1993), Bayesian Computation via the Gibbs Sampler and Related Markov Chain Monte Carlo Methods, Journal of the Royal Statistical Society B, 55, 3-23.

Stock, J. H. and M. W. Watson (2003), How Did Leading Indicator Forecasts Perform During the 2001 Recession?, Federal Reserve Bank of Richmond Economic Quarterly, 89, 71-90.

Swanson, N. R. and D. van Dijk (2006), Are Statistical Reporting Agencies Getting it Right? Data Rationality and Business Cycle Asymmetry, Journal of Business 8 Economic Statistics, 24, 24-42.

Tanner, M. A. and W. H. Wong (1987), The Calculation of Posterior Distributions by Data Augmentation, Journal of the American Statistical Association, 82, 528550 .

The Conference Board (2001), Business Cycle Indicators Handbook, The Conference Board, New York.

Tierney, L. (1994), Markov Chains for Exploring Posterior Distributions, The Annals of Statistics, 22, 1701-1762.

Zellner, A. (1971), An Introduction to Bayesian Inference in Econometrics, Wiley, New York. 
Table 1: Possible cycle interrelationships in bivariate Markov Switching model

Specification $\quad$ Graphical example

$\underline{\text { Independent cycles }}$

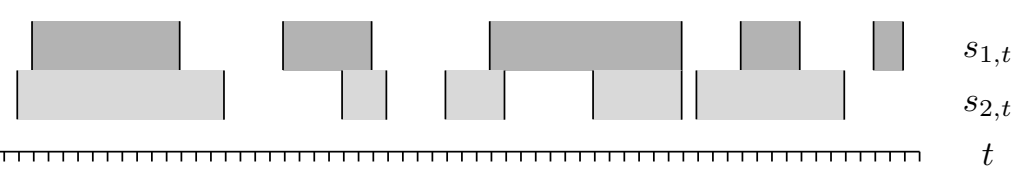

$\underline{\text { Synchronous common cycle }}$

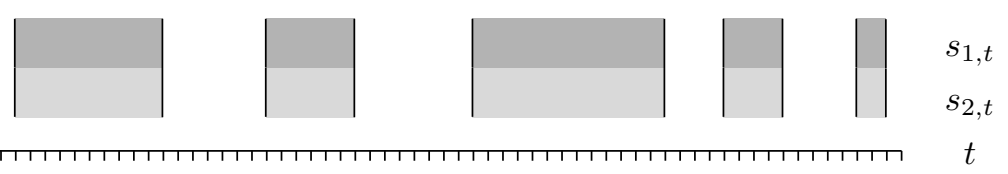

$\underline{\text { Non-synchronous common cycle with lead time } \kappa}$

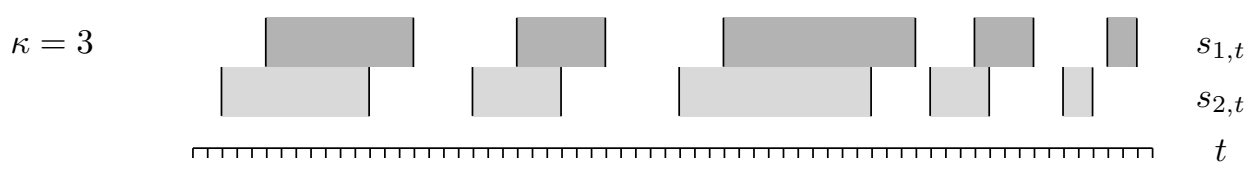

Non-synchronous common cycle with asymmetric lead times $\kappa_{1}$ for peaks and $\kappa_{2}$ for troughs

$$
\begin{aligned}
& \kappa_{1}=3 \\
& \kappa_{2}=5 \\
& \kappa_{1}=8 \\
& \kappa_{2}=5
\end{aligned}
$$
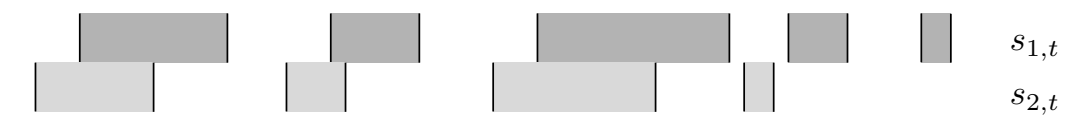

$s_{2, t}$
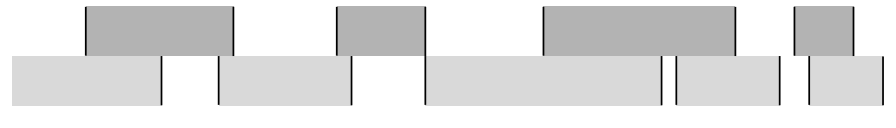

$s_{1, t}$

$s_{2, t}$

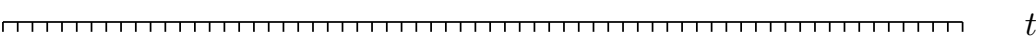

Note: The table shows four possible types of specifications for the processes $s_{1, t}$ and $s_{2, t}$ in the bivariate Markov Switching model (3), with different types of relationship between the cycles in $y_{1, t}$ and $y_{2, t}$ : (i) Independent cycles as implied by (5), (ii) A synchronous common cycle as in (6), (iii) A non-synchronous common cycle with identical lead/lag time $\kappa$ at peaks and troughs as in (7), and (iv) A non-synchronous common cycle with different lead/lag times $\kappa_{1}$ at peaks and $\kappa_{2}$ at troughs as in (8). The dark and light grey shaded areas correspond to recession periods in $y_{1, t}$ and $y_{2, t}$, respectively. 
Table 2: Posterior means and standard deviations (in parentheses) of parameters in linear and MS-VAR models without volatility break

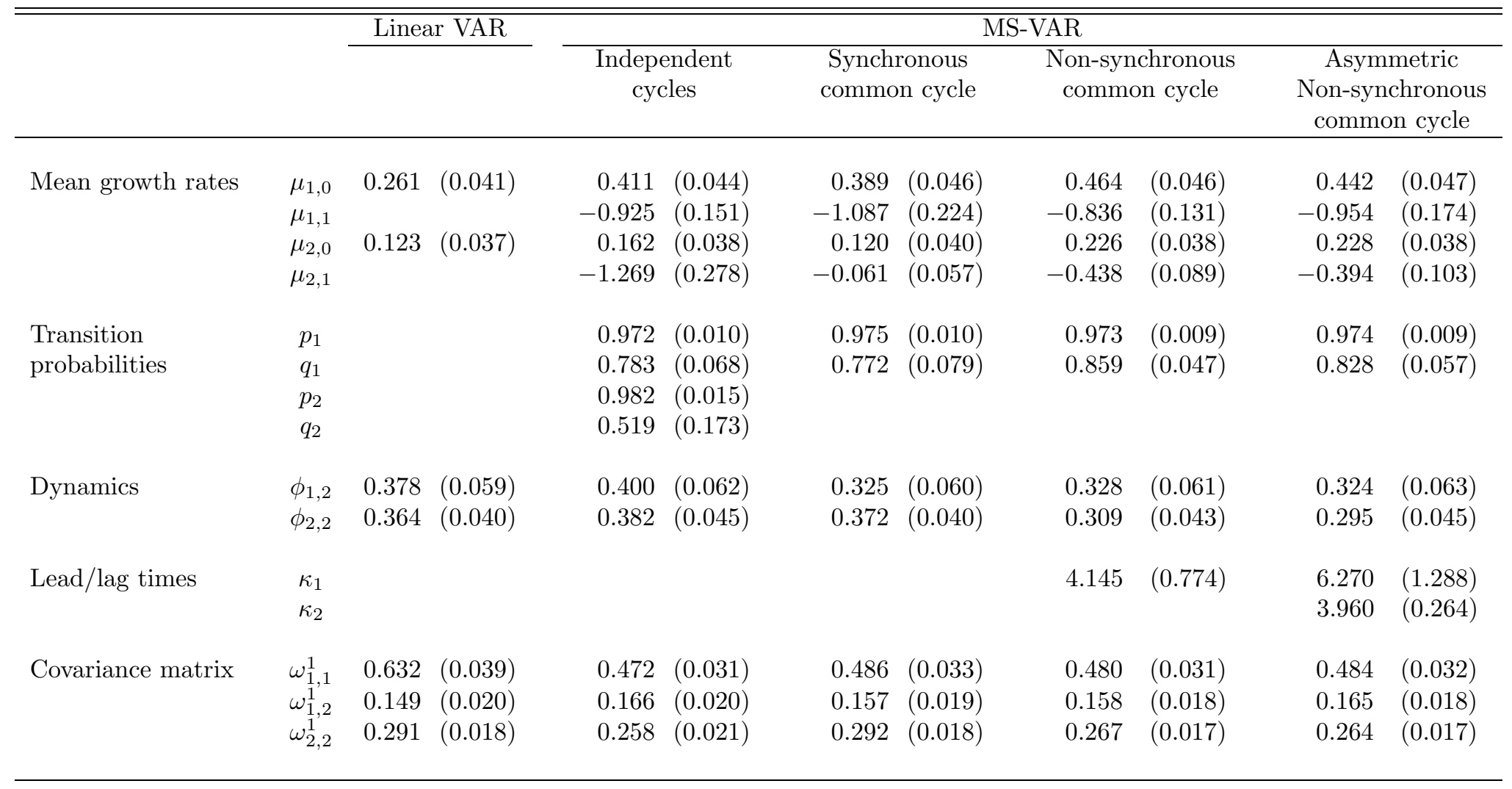

Log marg. likelihood

$-1045.6$

$-1007.5$

$-1021.1$

$-996.0$

$-988.3$

Note: The table presents posterior means and standard deviations (in parentheses) of parameters in linear and MS-VAR models, estimated for monthly growth rates of US IP and the Conference Board CLI over the period January 1959 - June 2004 . The four specifications for the processes $s_{1, t}$ and $s_{2, t}$ in the bivariate MS-VAR model are such that IP and the CLI have (i) independent cycles as implied by (5), (ii) a synchronous common cycle as in (6), (iii) a non-synchronous common cycle with identical lead/lag time $\kappa$ at peaks and troughs as in (7), and (iv) a non-synchronous common cycle with different lead/lag times $\kappa_{1}$ at peaks and $\kappa_{2}$ at troughs as in (8). Posterior results are based on 100,000 draws. Number of burn-in simulations is $10,000$. 
Table 3: Posterior means and standard deviations (in parentheses) of parameters in linear and MS-VAR models with volatility break

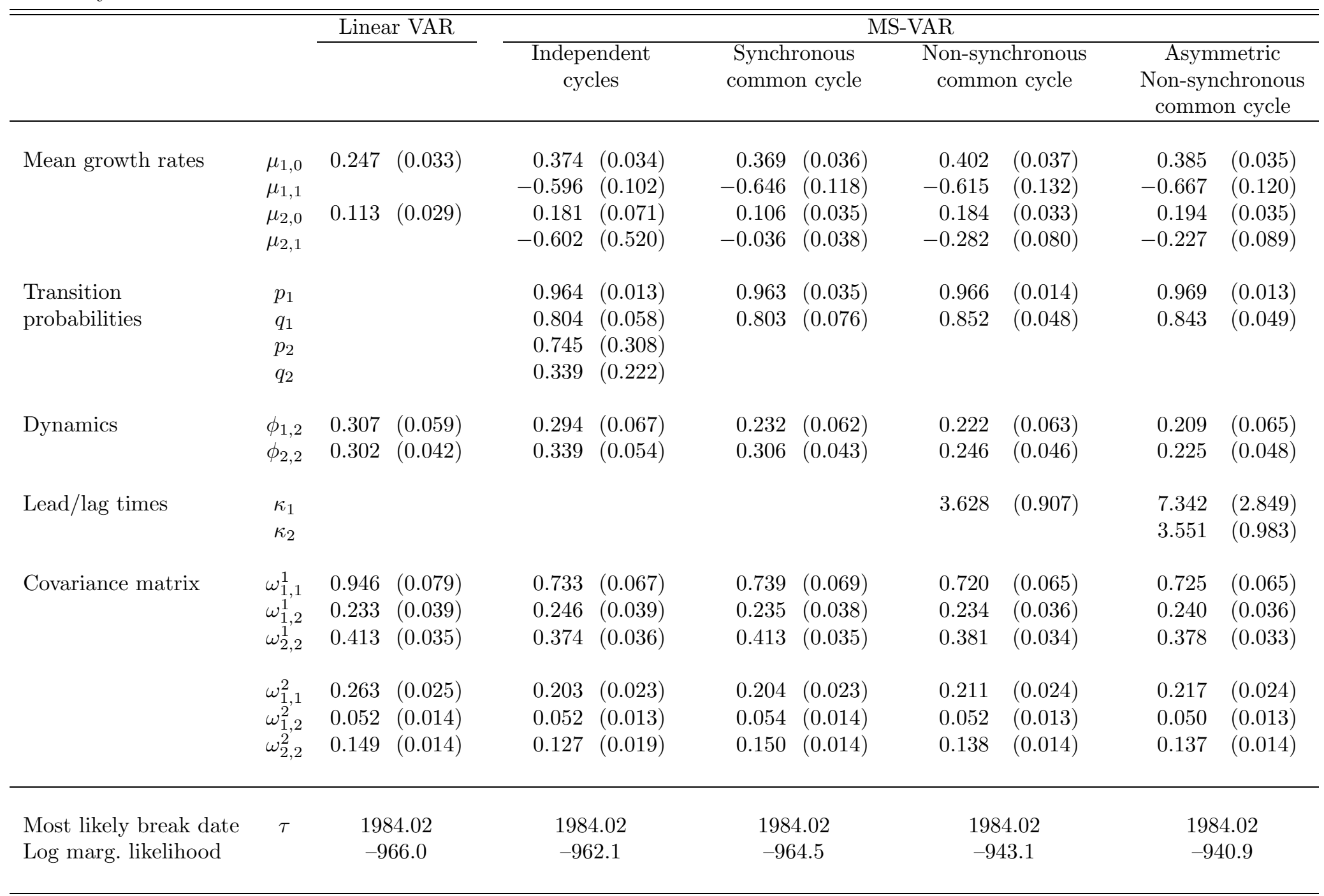

Note: The table presents posterior means and standard deviations (in parentheses) of parameters in linear and MS-VAR models with a single structural change in the covariance matrix as in (11), estimated for monthly growth rates of US IP and the Conference Board CLI over the period January 1959 - June 2004. The most likely break date is the mode of the posterior distribution of $\tau$. See Table 2 for definitions of the specifications for the processes $s_{1, t}$ and $s_{2, t}$ in the bivariate MS-VAR model. Posterior results are based on 100,000 draws. Number of burn-in simulations is 10,000 . 
Table 4: Diebold-Mariano (1995) tests

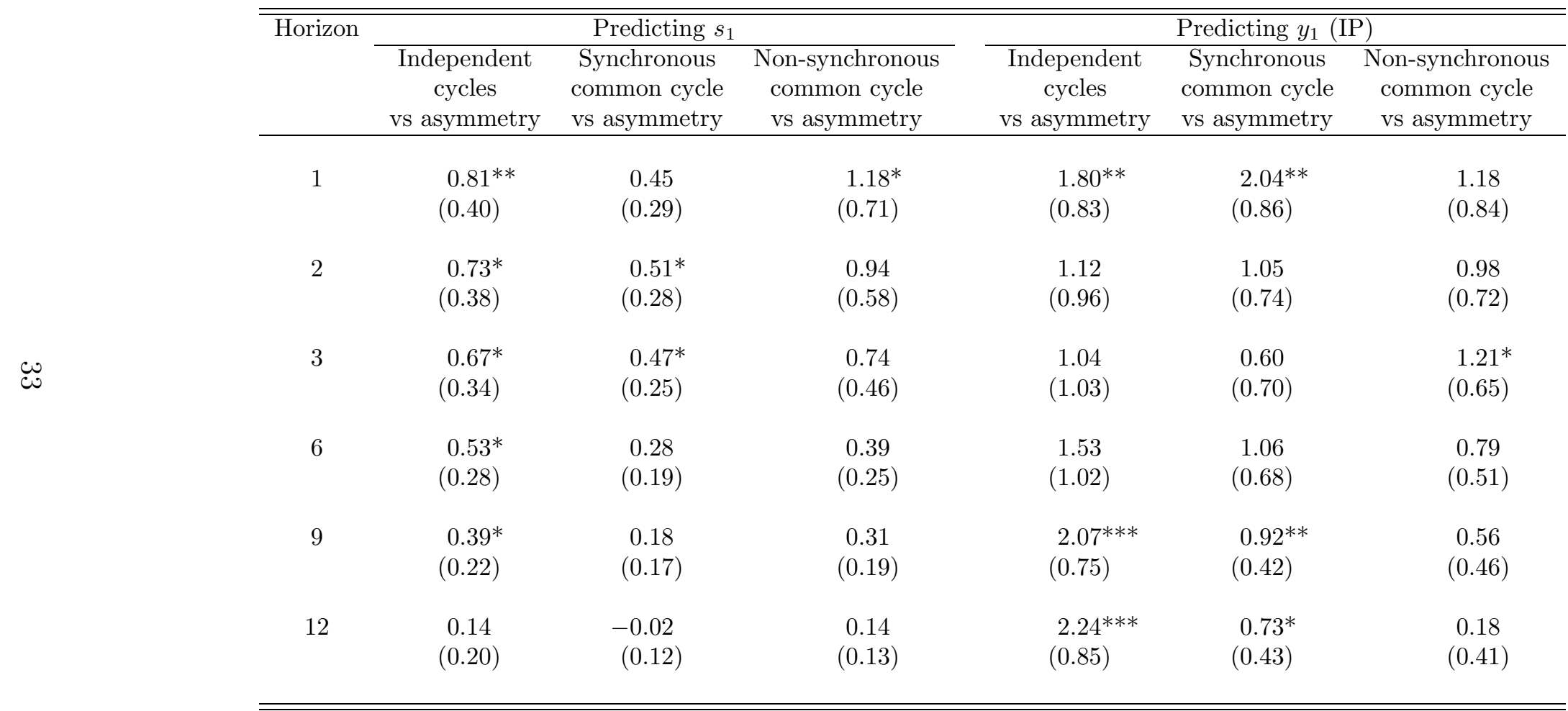

Heteroskedasticity and autocorrelation consistent (HAC) standard errors in parentheses.

All numbers are $\times 100$.

$* * *, * *$ and $*$ denotes significant at the $1 \%, 5 \%$ and $10 \%$ level of singificance, respectively. 
Figure 1: Time series plots of US Industrial Production (dashed lines) and the Composite Leading Index (solid lines)

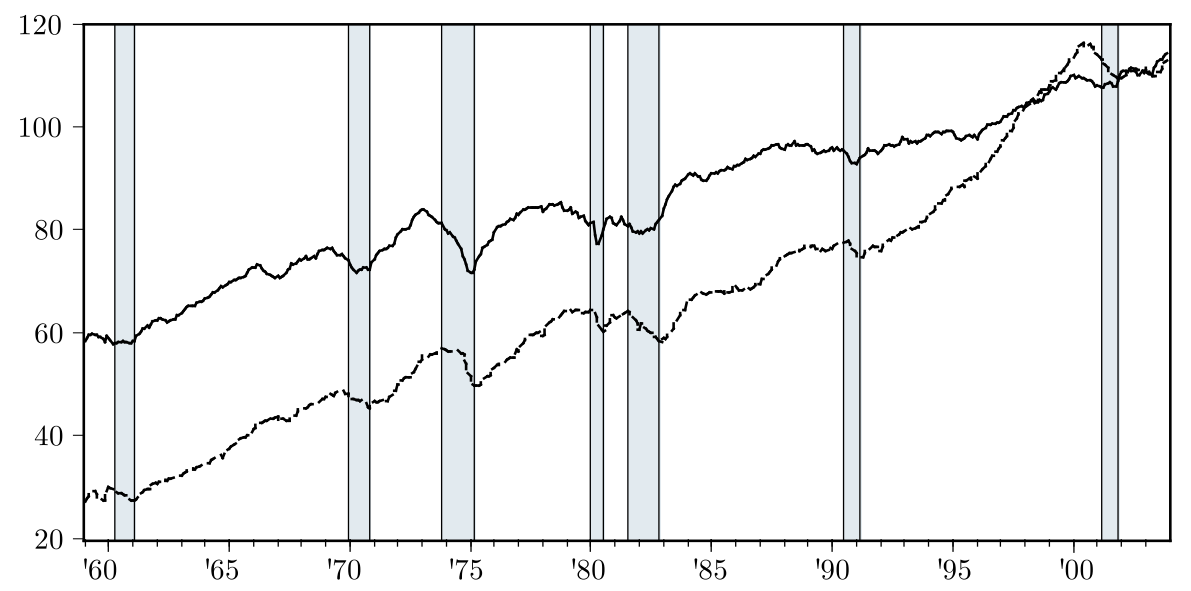

(a) Log levels

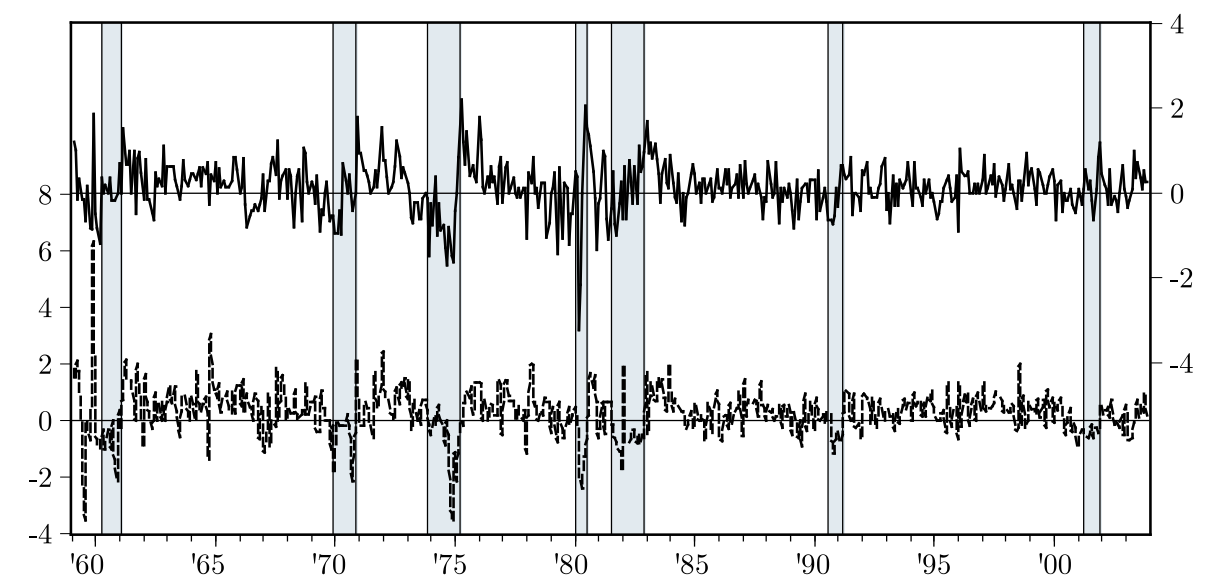

(b) Percentage growth rates 
Figure 2: Smoothed recession probabilities in MS-VAR models without volatility break (a) NBER turning points

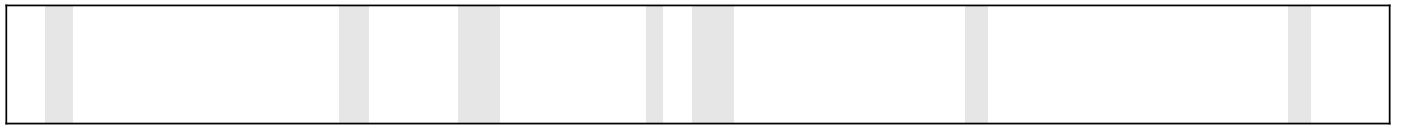

(b) Independent cycles

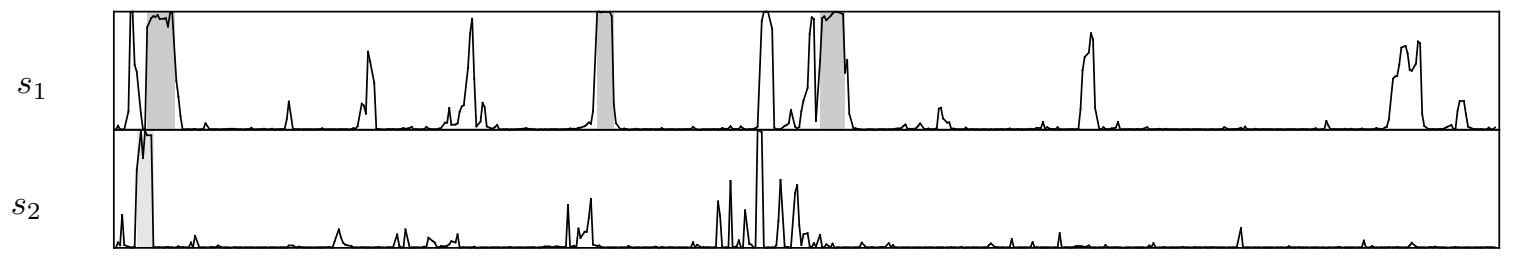

(c) Synchronous common cycle

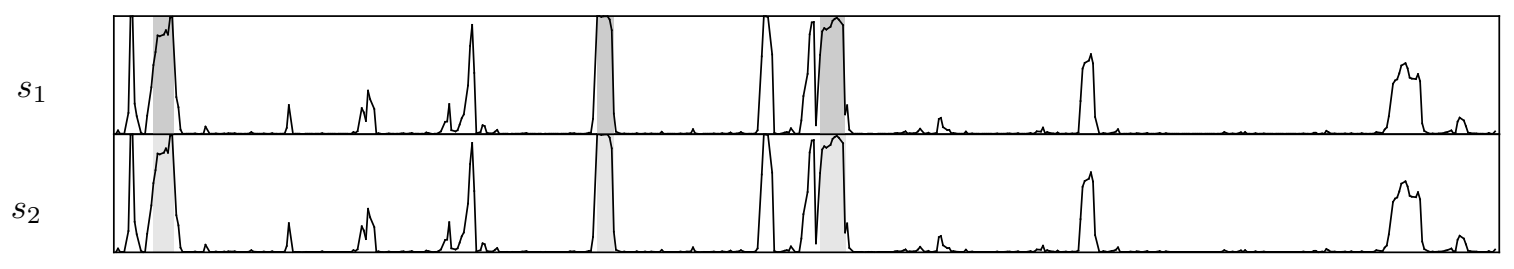

(d) Non-synchronous common cycle

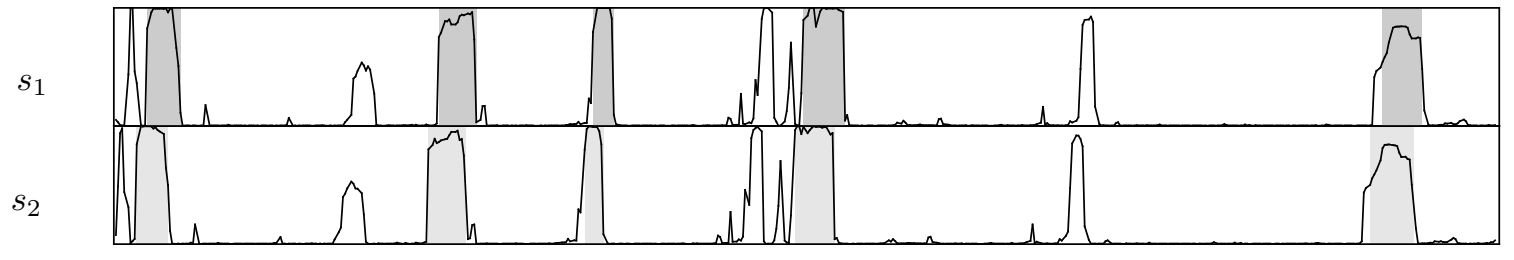

(e) Non-synchronous common cycle with asymmetric lead times for peaks and troughs

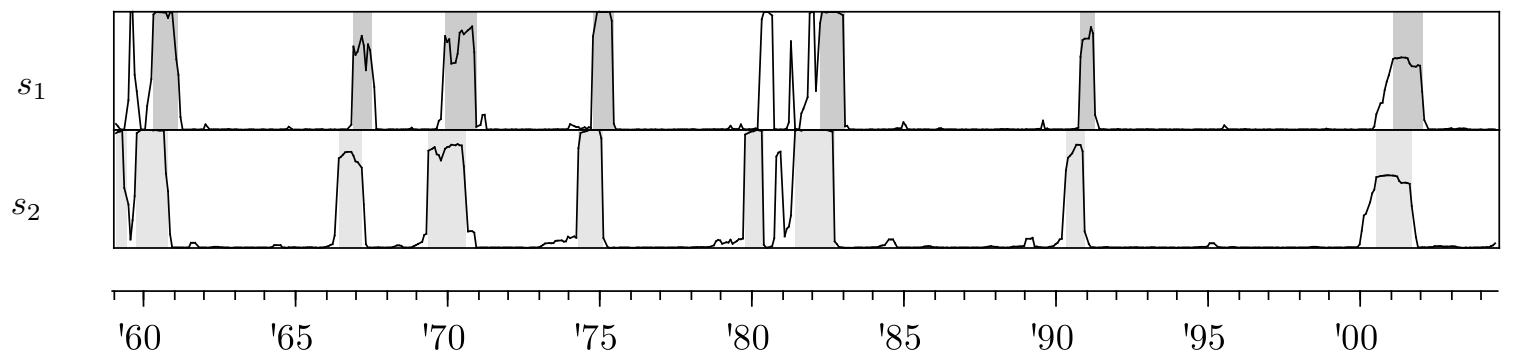

Note: The graphs present smoothed recession probabilities in MS-VAR models estimated for monthly growth rates of US IP and the Conference Board CLI over the period January 1959 June 2004, with different types of relationships between their cycles. See Table 2 for definitions of the specifications for the processes $s_{1, t}$ and $s_{2, t}$. The dark and light grey shaded areas correspond to recessions in IP and the CLI, respectively, following the rule that a recession is defined as (at least) six consecutive months where the posterior mean of $s_{j, t}$ is larger than 0.5 . 
Figure 3: Posterior density of the variance breakpoint parameter $\tau$

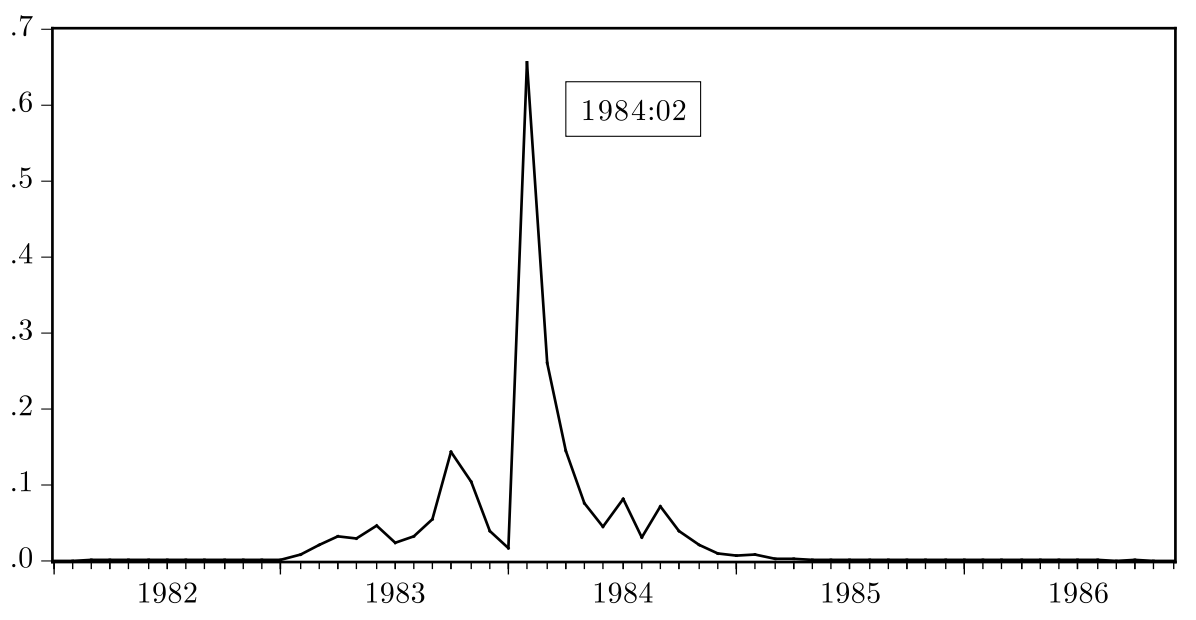

Note: The graph presents the posterior density of the variance break date $\tau$ (for the period January 1982 - December 1986) in the MS-VAR model with a non-synchronous common cycle with different lead/lag times $\kappa_{1}$ at peaks and $\kappa_{2}$ at troughs as in (8) and a single structural change in the covariance matrix as in (11), estimated for monthly growth rates of US IP and the Conference Board CLI over the period January 1959 - June 2004.

Figure 4: Joint posterior density of the lead/lag parameters $\kappa_{1}$ and $\kappa_{2}$

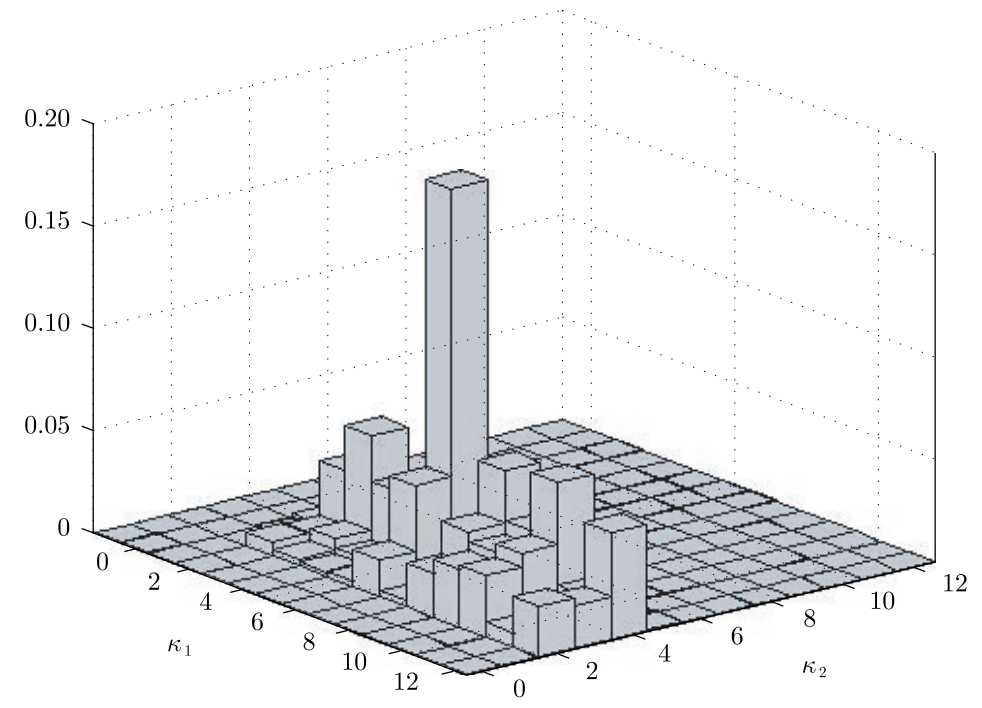

Note: The graph presents the joint posterior density of the lead/lag parameters $\kappa_{1}$ and $\kappa_{2}$ in the MS-VAR model with a non-synchronous common cycle with asymmetric lead/lag times $\kappa_{1}$ at peaks and $\kappa_{2}$ at troughs as in (8) and a single structural change in the covariance matrix as in (11), estimated for monthly growth rates of US IP and the Conference Board CLI over the period January 1959 - June 2004. 
Figure 5: Smoothed recession probabilities in MS-VAR models with volatility break (a) NBER turning points

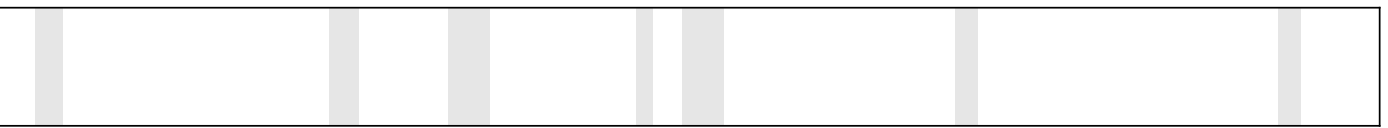

(b) Independent cycles

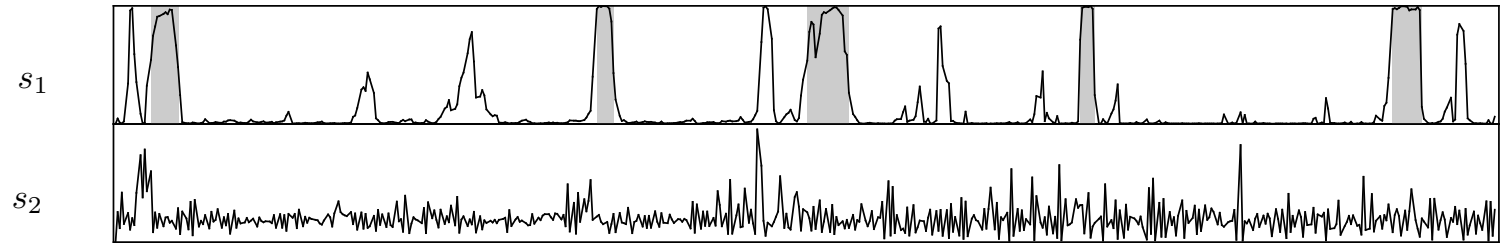

(c) Synchronous common cycle

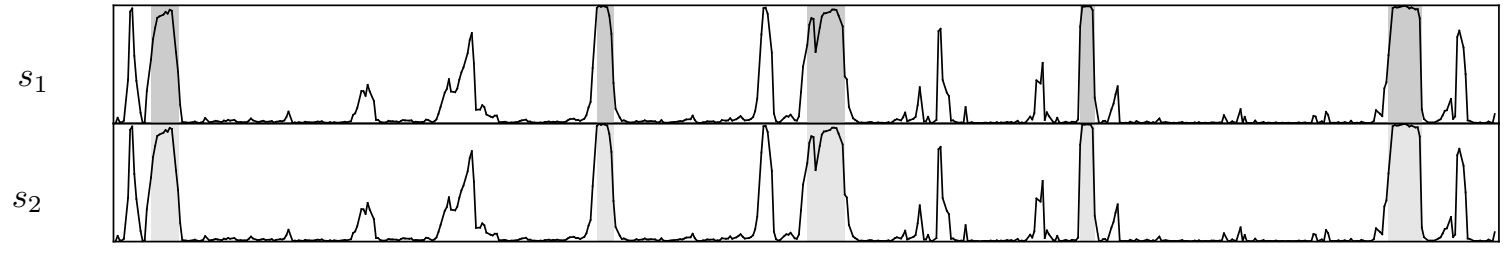

(d) Non-synchronous common cycle

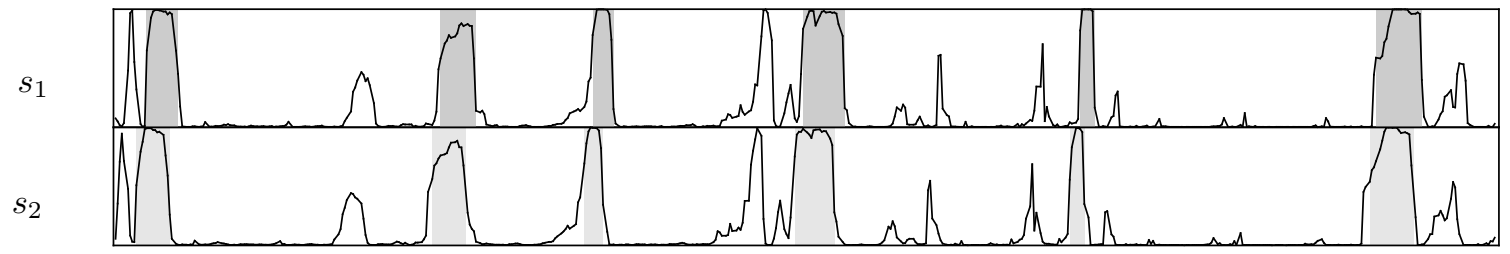

(e) Non-synchronous common cycle with asymmetric lead times for peaks and troughs

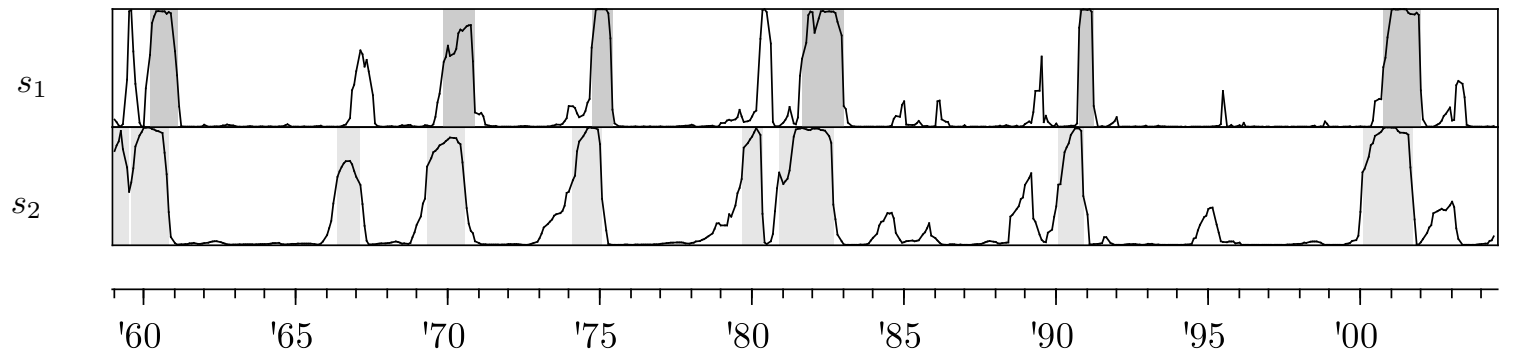

Note: The graphs present smoothed recession probabilities in MS-VAR models with a single structural change in the covariance matrix as in (11) estimated for monthly growth rates of US IP and the Conference Board CLI over the period January 1959 - June 2004, with different types of relationships between their cycles. See Table 2 for definitions of the specifications for the processes $s_{1, t}$ and $s_{2, t}$. The dark and light grey shaded areas correspond to recessions in IP and the CLI, respectively, following the rule that a recession is defined as (at least) six consecutive months where the posterior mean of $s_{j, t}$ is larger than 0.5. 
Figure 6: In-sample estimates and out-of-sample predictions of recession probabilities in a rolling horizon: The July 1990 - March 1991 recession

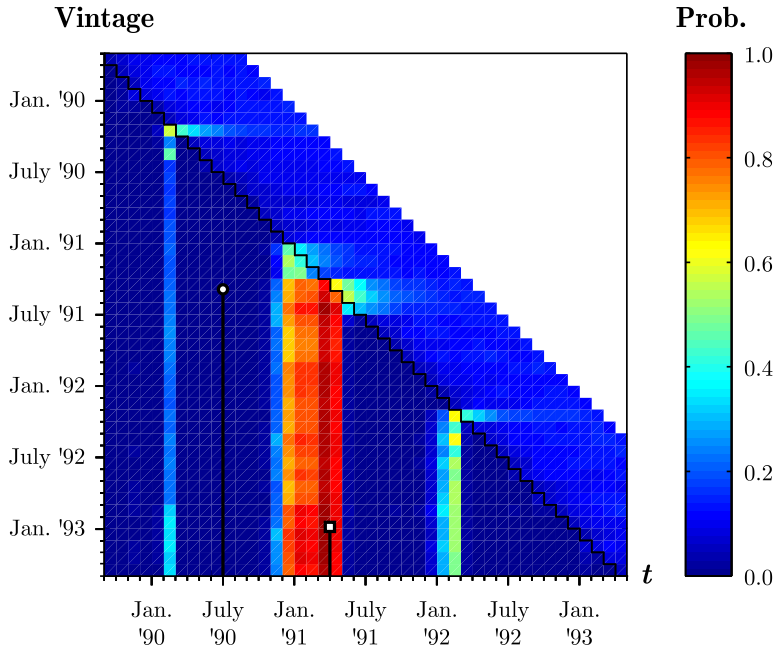

(a) Independent cycles

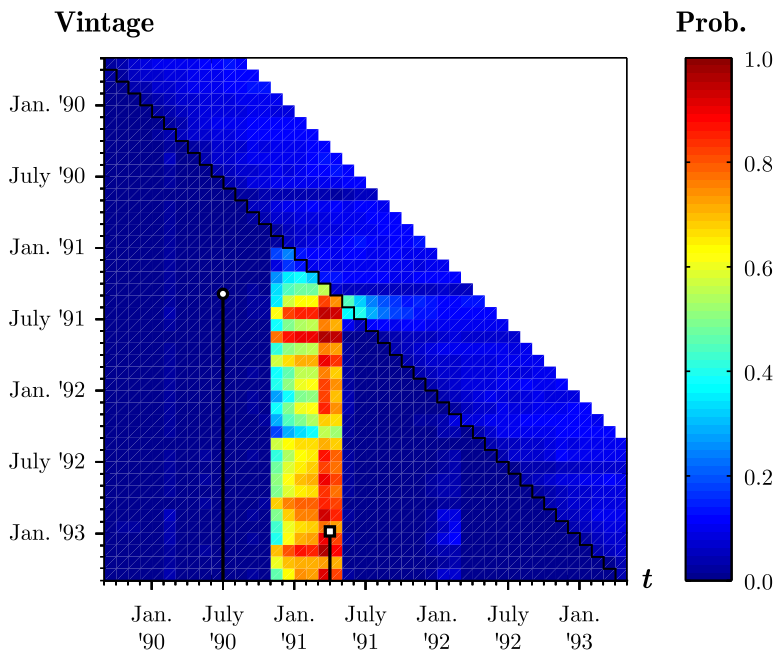

(c) Non-synchronous common cycle

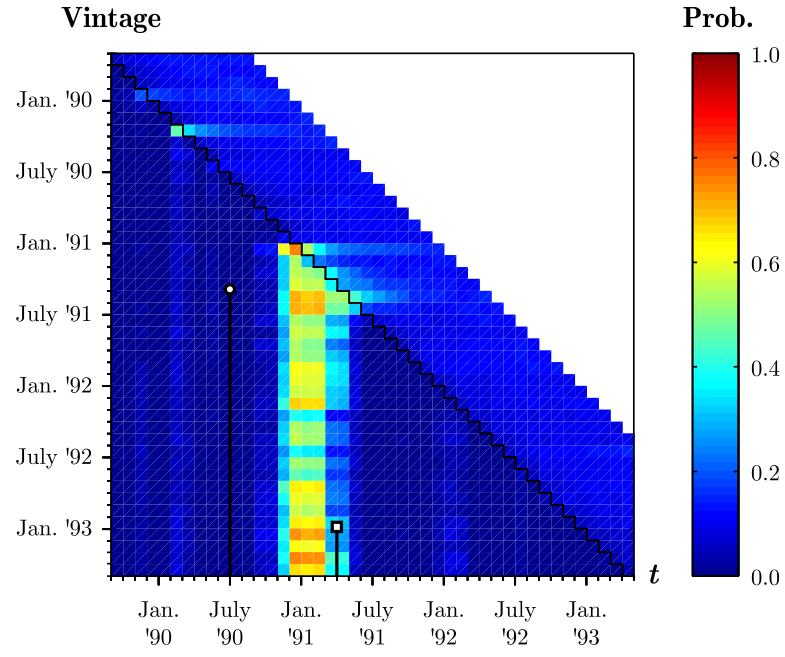

(b) Synchronous common cycle

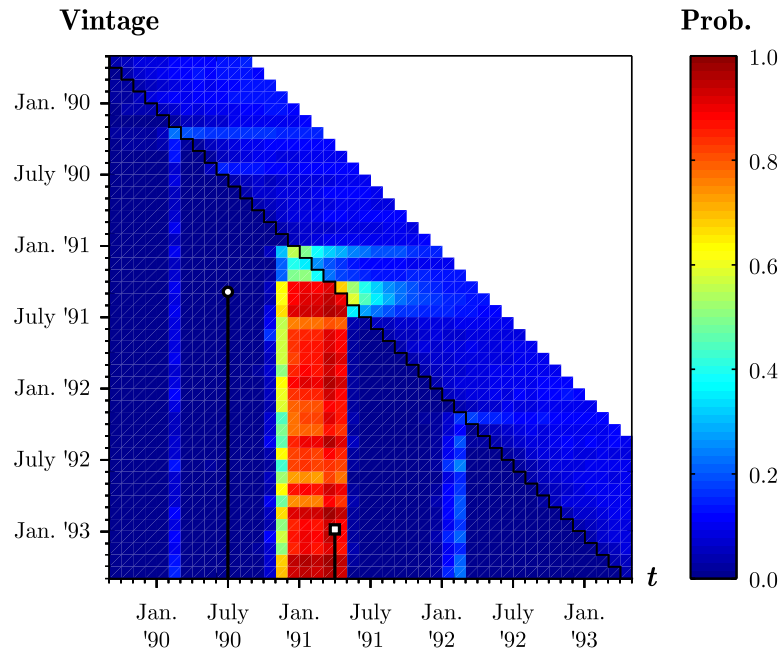

(d) Asymmetric non-synchronous common cycle

Note: The graph presents the estimated and predicted recession probabilities in a rolling horizon, where at every point in time the latest data vintage is used to compute in-sample estimates for the past and out-of-sample predictions for the next 12 months ahead. The in-sample estimates and out-of-sample predictions are separated by the stepwise diagonal line. The announcement date of the July 1990 business cycle peak (April 25, 1991), is marked by the circle. Likewise, the square marks the announcement date of the March 1991 business cycle trough (December 22, 1992). 
Figure 7: In-sample estimates and out-of-sample predictions of recession probabilities in a rolling horizon: The March - November 2001 recession

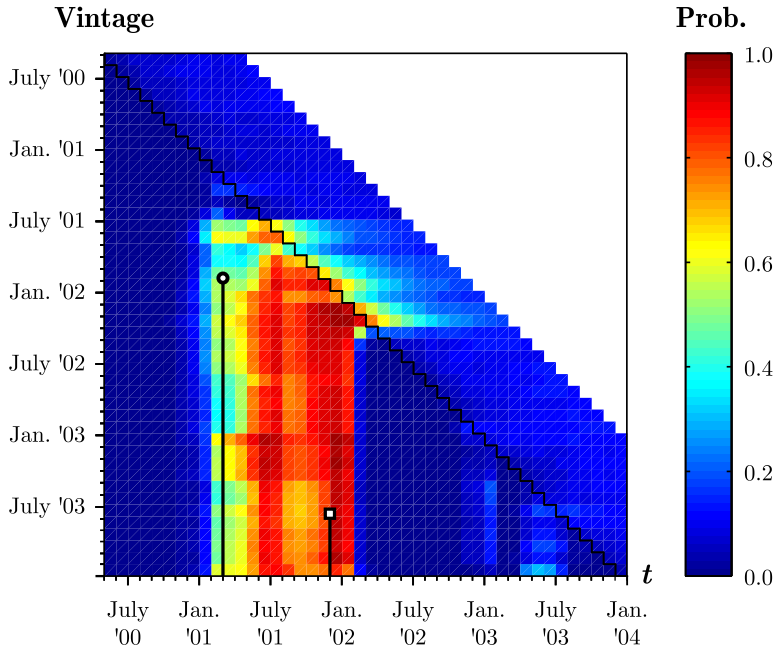

(a) Independent cycles

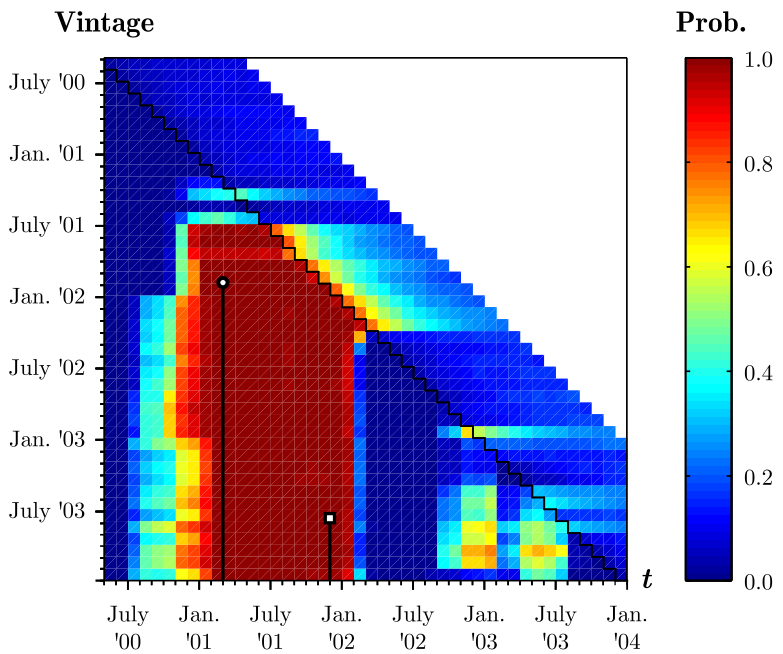

(c) Non-synchronous common cycle

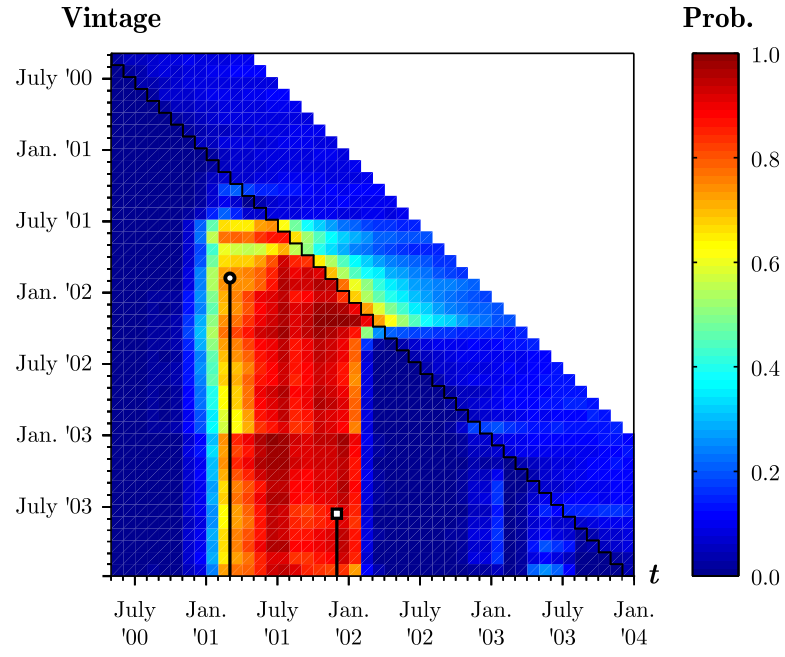

(b) Synchronous common cycle

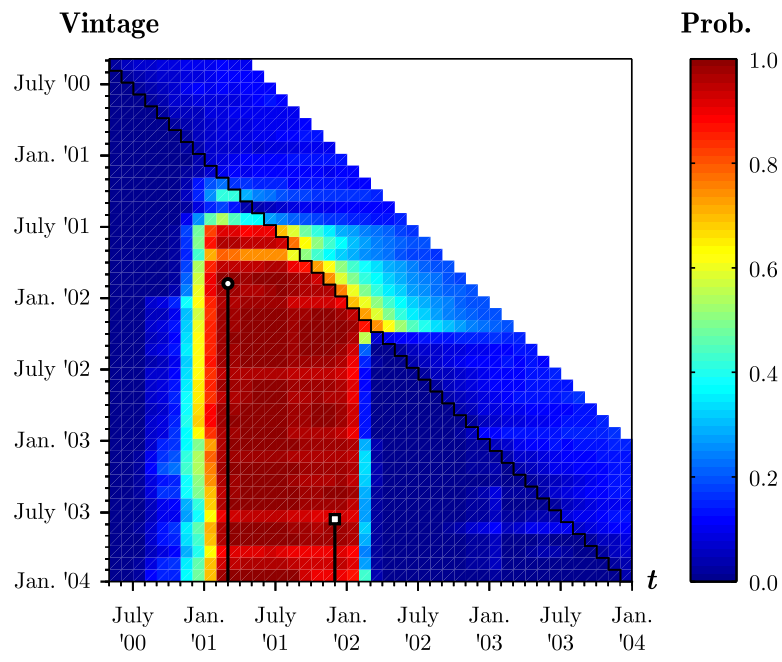

(d) Asymmetric non-synchronous common cycle

Note: The graph presents the estimated and predicted recession probabilities in a rolling horizon, where at every point in time the latest data vintage is used to compute in-sample estimates for the past and out-of-sample predictions for the next 12 months ahead. The in-sample estimates and out-of-sample predictions are separated by the stepwise diagonal line. The announcement date of the March 2001 business cycle peak (November 26, 2001), is marked by the circle. Likewise, the square marks the announcement date of the November 2001 business cycle trough (July 17, 2003). 
Figure 8: Regime and industrial production prediction error ratios

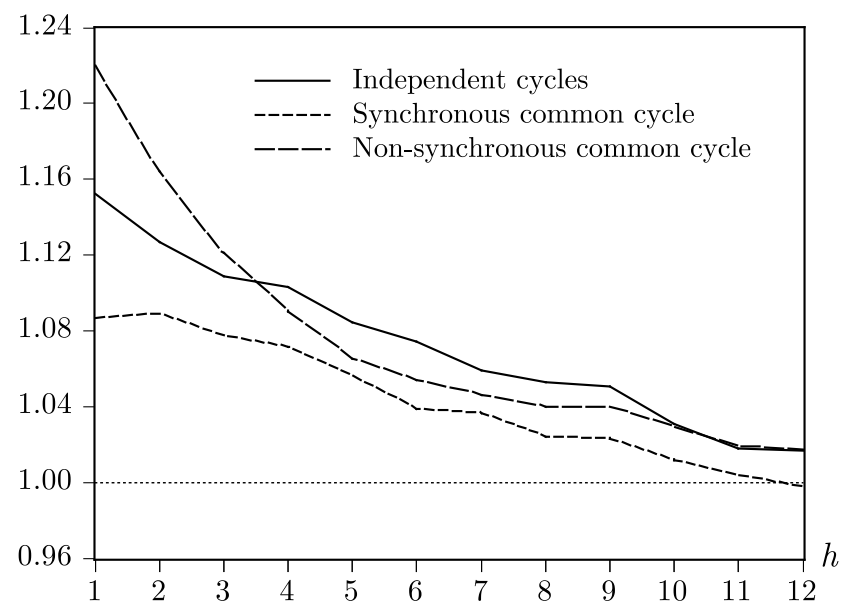

(a) Regime

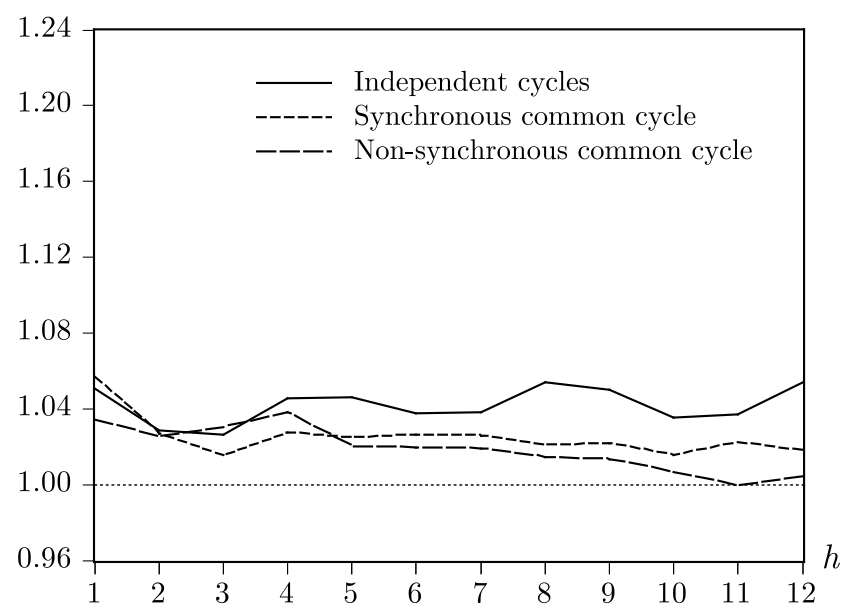

(b) Industrial production

Note: The graphs present forecasting error ratios obtained from the MS-VAR models with independent cycles, with a synchronous common cycle and with a symmetric non-synchronous common cycle relative to the asymmetric non-synchronous common cycle model. In panel (a), the TPFE $(h)$ for forecasts of the recession probabilities are shown, where $h=1,2, \ldots, 12$. Panel (b) shows the $\operatorname{MSFE}(h)$ for forecasts of IP growth. 